\title{
Routing Techniques in Wireless Sensor Networks: A SURVEY*
}

\author{
Jamal N. Al-Karaki Ahmed E. Kamal \\ Dept. of Electrical and Computer Engineering \\ Iowa State University, Ames, Iowa 50011 \\ Email: \{jkaraki, kamal\}@iastate.edu
}

\begin{abstract}
Wireless Sensor Networks (WSNs) consist of small nodes with sensing, computation, and wireless communications capabilities. Many routing, power management, and data dissemination protocols have been specifically designed for WSNs where energy awareness is an essential design issue. The focus, however, has been given to the routing protocols which might differ depending on the application and network architecture. In this paper, we present a survey of the state-of-the-art routing techniques in WSNs. We first outline the design challenges for routing protocols in WSNs followed by a comprehensive survey of different routing techniques. Overall, the routing techniques are classified into three categories based on the underlying network structure: flat, hierarchical, and location-based routing. Furthermore, these protocols can be classified into multipath-based, query-based, negotiation-based, QoS-based, and coherent-based depending on the protocol operation. We study the design tradeoffs between energy and communication overhead savings in every routing paradigm. We also highlight the advantages and performance issues of each routing technique. The paper concludes with possible future research areas.
\end{abstract}

\section{Introduction}

Due to recent technological advances, the manufacturing of small and low cost sensors became technically and economically feasible. The sensing electronics measure ambient conditions related to the environment surrounding the sensor and transforms them into an electric signal. Processing such a signal reveals some properties about objects located and/or events happening in the vicinity of the sensor. A large number of these disposable sensors can be networked in many applications that require unattended operations. A Wireless Sensor Network (WSN) contain hundreds or thousands of these sensor nodes. These sensors have the ability to communicate either among each other or directly to an external base-station (BS). A greater number of sensors allows for sensing over larger geographical regions with greater accuracy. Figure 1 shows the schematic diagram of sensor node components. Basically, each sensor node comprises sensing, processing, transmission, mobilizer, position finding system, and power units (some of these components are optional like the mobilizer). The same figure shows the communication architecture of a WSN. Sensor nodes are usually scattered in a sensor field, which is an area where the sensor nodes are deployed. Sensor nodes coordinate among themselves to produce high-quality information about the physical environment. Each sensor node bases its decisions on its mission, the information it currently has, and its knowledge of its

\footnotetext{
${ }^{*}$ This research was supported in part by the ICUBE initiative of Iowa State University, Ames, IA 50011.
} 
computing, communication, and energy resources. Each of these scattered sensor nodes has the capability to collect and route data either to other sensors or back to an external base station(s) ${ }^{1}$. A base-station may be a fixed node or a mobile node capable of connecting the sensor network to an existing communications infrastructure or to the Internet where a user can have access to the reported data.

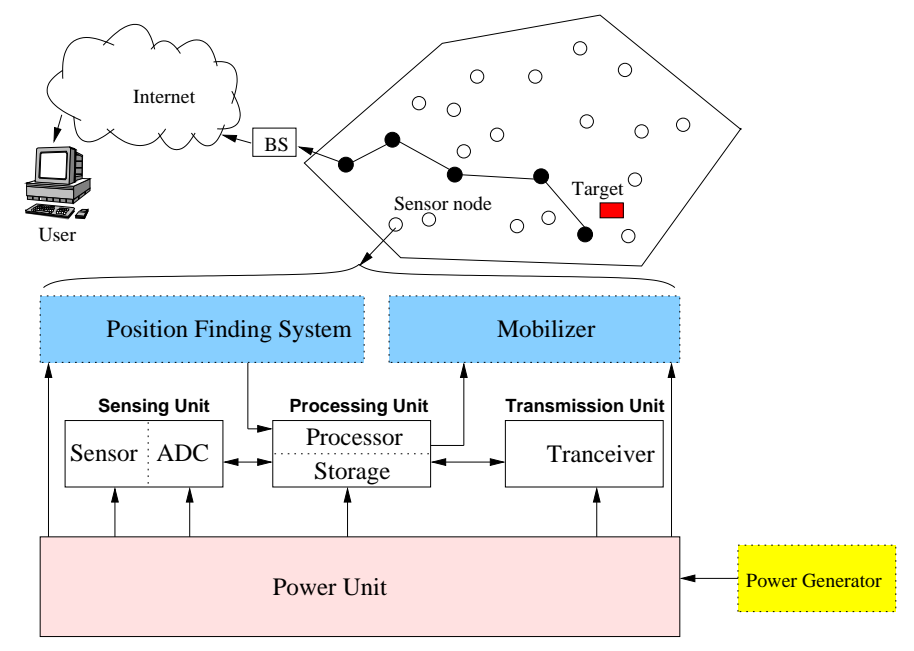

Figure 1: The components of a sensor node

Networking unattended sensor nodes may have profound effect on the efficiency of many military and civil applications such as target field imaging, intrusion detection, weather monitoring, security and tactical surveillance, distributed computing, detecting ambient conditions such as temperature, movement, sound, light, or the presence of certain objects, inventory control, and disaster management. Deployment of a sensor network in these applications can be in random fashion (e.g., dropped from an airplane) or can be planted manually (e.g., fire alarm sensors in a facility). For example, in a disaster management application, a large number of sensors can be dropped from a helicopter. Networking these sensors can assist rescue operations by locating survivors, identifying risky areas, and making the rescue team more aware of the overall situation in the disaster area.

In the past few years, an intensive research that addresses the potential of collaboration among sensors in data gathering and processing and in the coordination and management of the sensing activity were conducted. However, sensor nodes are constrained in energy supply and bandwidth. Thus, innovative techniques that eliminate energy inefficiencies that would shorten the lifetime of the network are highly required. Such constraints combined with a typical deployment of large number of sensor nodes pose many challenges to the design and management of WSNs and necessitiate energy-awareness at all layers of the networking protocol stack. For example, at the network layer, it is highly desirable to find methods for energy-efficient route discovery and relaying of data from the sensor nodes to the BS so that the lifetime of the network is maximized.

Routing in WSNs is very challenging due to the inherent characteristics that distinguish these networks from other wireless networks like mobile ad hoc networks or cellular networks. First, due to the relatively large number of sensor nodes, it is not possible to build a global addressing scheme for the deployment of a large number of sensor nodes as the overhead of ID maintenance is high. Thus, traditional IP-based protocols may not be applied to WSNs. Furthermore, sensor nodes that are deployed in an ad hoc manner

\footnotetext{
${ }^{1}$ In this paper, we consider routing towards a BS only
} 
need to be self-organizing as the ad hoc deployment of these nodes requires the system to form connections and cope with the resultant nodal distribution especially that the operation of the sensor networks is unattended. In WSNs, sometimes getting the data is more important than knowing the IDs of which nodes sent the data. Second, in contrast to typical communication networks, almost all applications of sensor networks require the flow of sensed data from multiple sources to a particular BS. This, however, does not prevent the flow of data to be in other forms (e.g., multicast or peer to peer). Third, sensor nodes are tightly constrained in terms of energy, processing, and storage capacities. Thus, they require careful resource management. Fourth, in most application scenarios, nodes in WSNs are generally stationary after deployment except for, may be, a few mobile nodes. Nodes in other traditional wireless networks are free to move, which results in unpredictable and frequent topological changes. However, in some applications, some sensor nodes may be allowed to move and change their location (although with very low mobility). Fourth, sensor networks are application specific, i.e., design requirements of a sensor network change with application. For example, the challenging problem of low-latency precision tactical surveillance is different from that required for a periodic weather-monitoring task. Fifth, position awareness of sensor nodes is important since data collection is normally based on the location. Currently, it is not feasible to use Global Positioning System (GPS) hardware for this purpose. Methods based on triangulation [20], for example, allow sensor nodes to approximate their position using radio strength from a few known points. It is found in [20] that algorithms based on triangulation or multilateration can work quite well under conditions where only very few nodes know their positions apriori, e.g., using GPS hardware. Still, it is favorable to have GPS-free solutions [21] for the location problem in WSNs. Finally, data collected by many sensors in WSNs is typically based on common phenomena, hence there is a high probability that this data has some redundancy. Such redundancy needs to be exploited by the routing protocols to improve energy and bandwidth utilization. Usually, WSNs are data-centric networks in the sense that data is requested based on certain attributes, i.e., attribute-based addressing. An attribute-based address is composed of a set of attribute-value pair query. For example, if the query is something like [temperature $>60 \mathrm{~F}$ ], then sensor nodes that sense temperature $>60 \mathrm{~F}$ only need to respond and report their readings.

Due to such differences, many new algorithms have been proposed for the routing problem in WSNs. These routing mechanisms have taken into consideration the inherent features of WSNs along with the application and architecture requirements. The task of finding and maintaining routes in WSNs is nontrivial since energy restrictions and sudden changes in node status (e.g., failure) cause frequent and unpredictable topological changes. To minimize energy consumption, routing techniques proposed in the literature for WSNs employ some well-known routing tactics as well as tactics special to WSNs, e.g., data aggregation and in-network processing, clustering, different node role assignment, and data-centric methods were employed. Almost all of the routing protocols can be classified according to the network structure as flat, hierarchical, or location-based. Furthermore, these protocols can be classified into multipath-based, query-based, negotiation-based, QoS-based, and coherent-based depending on the protocol operation. In flat networks, all nodes play the same role while hierarchical protocols aim at clustering the nodes so that cluster heads can do some aggregation and reduction of data in order to save energy. Location-based protocols utilize the position information to relay the data to the desired regions rather than the whole network. The last category includes routing approaches that are based on the protocol operation, which vary according to the approach used in the protocol. In this paper, we explore these routing techniques in WSNs that have been developed in recent years and develop a classification for these protocols. Then, we discuss each of 
the routing protocols under this classification. Our objective is to provide deeper understanding of the current routing protocols in WSNs and identify some open research issues that can be further pursued.

Although there are some previous efforts for surveying the characteristics, applications, and communication protocols in WSNs $[4,37$, the scope of the survey presented in this paper is distinguished from these surveys in many aspects. The surveys in [4] and [37] addressed several design issues and techniques for WSNs describing the physical constraints on sensor nodes, applications, architectural attributes, and the protocols proposed in all layers of the network stack. However, these surveys were not devoted to routing only. Due to the importance of routing in WSNs and the availability of a significant body of literature on this topic, a detailed survey becomes necessary and useful at this stage. Our work is a dedicated study of the network layer, describing and categorizing the different approaches for data routing. In addition, we summarize routing challenges and design issues that may affect the performance of routing protocols in WSNs. The rest of this paper is organized as follows. In Section 2, we discuss routing challenges and design issues in WSNs. A classification and a comprehensive survey of routing techniques in WSNs is presented in Section 3. In Section 4, a summary of future research directions on routing in WSNs is discussed. We conclude with final remarks in Section 5.

\section{Routing Challenges and Design Issues in WSNs}

Despite the innumerable applications of WSNs, these networks have several restrictions, e.g., limited energy supply, limited computing power, and limited bandwidth of the wireless links connecting sensor nodes. One of the main design goals of WSNs is to carry out data communication while trying to prolong the lifetime of the network and prevent connectivity degradation by employing aggressive energy management techniques. The design of routing protocols in WSNs is influenced by many challenging factors. These factors must be overcome before efficient communication can be achieved in WSNs. In the following, we summarize some of the routing challenges and design issues that affect routing process in WSNs.

- Node deployment: Node deployment in WSNs is application dependent and affects the performance of the routing protocol. The deployment can be either deterministic or randomized. In deterministic deployment, the sensors are manually placed and data is routed through pre-determined paths. However, in random node deployment, the sensor nodes are scattered randomly creating an infrastructure in an ad hoc manner. If the resultant distribution of nodes is not uniform, optimal clustering becomes necessary to allow connectivity and enable energy efficient network operation. Inter-sensor communication is normally within short transmission ranges due to energy and bandwidth limitations. Therefore, it is most likely that a route will consist of multiple wireless hops.

- Energy consumption without losing accuracy: sensor nodes can use up their limited supply of energy performing computations and transmitting information in a wireless environment. As such, energyconserving forms of communication and computation are essential. Sensor node lifetime shows a strong dependence on the battery lifetime [1]. In a multihop WSN, each node plays a dual role as data sender and data router. The malfunctioning of some sensor nodes due to power failure can cause significant topological changes and might require rerouting of packets and reorganization of the network. 
- Data Reporting Model: Data sensing and reporting in WSNs is dependent on the application and the time criticality of the data reporting. Data reporting can be categorized as either time-driven (continuous), event-driven, query-driven, and hybrid [13]. The time-driven delivery model is suitable for applications that require periodic data monitoring. As such, sensor nodes will periodically switch on their sensors and transmitters, sense the environment and transmit the data of interest at constant periodic time intervals. In event-driven and query-driven models, sensor nodes react immediately to sudden and drastic changes in the value of a sensed attribute due to the occurrence of a certain event or a query is generated by the BS. As such, these are well suited for time critical applications. A combination of the previous models is also possible. The routing protocol is highly influenced by the data reporting model with regard to energy consumption and route stability.

- Node/Link Heterogeneity: In many studies, all sensor nodes were assumed to be homogeneous, i.e., having equal capacity in terms of computation, communication, and power. However, depending on the application a sensor node can have different role or capability. The existence of heterogeneous set of sensors raises many technical issues related to data routing. For example, some applications might require a diverse mixture of sensors for monitoring temperature, pressure and humidity of the surrounding environment, detecting motion via acoustic signatures, and capturing the image or video tracking of moving objects. These special sensors can be either deployed independently or the different functionalities can be included in the same sensor nodes. Even data reading and reporting can be generated from these sensors at different rates, subject to diverse quality of service constraints, and can follow multiple data reporting models. For example, hierarchical protocols designate a clusterhead node different from the normal sensors. These clusterheads can be chosen from the deployed sensors or can be more powerful than other sensor nodes in terms of energy, bandwidth, and memory. Hence, the burden of transmission to the BS is handled by the set of cluster-heads.

- Fault Tolerance: Some sensor nodes may fail or be blocked due to lack of power, physical damage, or environmental interference. The failure of sensor nodes should not affect the overall task of the sensor network. If many nodes fail, MAC and routing protocols must accommodate formation of new links and routes to the data collection base stations. This may require actively adjusting transmit powers and signaling rates on the existing links to reduce energy consumption, or rerouting packets through regions of the network where more energy is available. Therefore, multiple levels of redundancy may be needed in a fault-tolerant sensor network.

- Scalability: The number of sensor nodes deployed in the sensing area may be in the order of hundreds or thousands, or more. Any routing scheme must be able to work with this huge number of sensor nodes. In addition, sensor network routing protocols should be scalable enough to respond to events in the environment. Until an event occurs, most of the sensors can remain in the sleep state, with data from the few remaining sensors providing a coarse quality.

- Network Dynamics: Most of the network architectures assume that sensor nodes are stationary. However, mobility of both BS's or sensor nodes is sometimes necessary in many applications [19]. Routing messages from or to moving nodes is more challenging since route stability becomes an important issue, in addition to energy, bandwidth etc. Moreover, the sensed phenomenon can be either dynamic or static depending on the application, e.g., it is dynamic in a target detection/tracking application, 
while it is static in forest monitoring for early fire prevention. Monitoring static events allows the network to work in a reactive mode, simply generating traffic when reporting. Dynamic events in most applications require periodic reporting and consequently generate significant traffic to be routed to the BS.

- Transmission Media: In a multi-hop sensor network, communicating nodes are linked by a wireless medium. The traditional problems associated with a wireless channel (e.g., fading, high error rate) may also affect the operation of the sensor network. In general, the required bandwidth of sensor data will be low, on the order of 1-100 kb/s. Related to the transmission media is the design of medium access control (MAC). One approach of MAC design for sensor networks is to use TDMA based protocols that conserve more energy compared to contention based protocols like CSMA (e.g., IEEE 802.11). Bluetooth technology [32] can also be used.

- Connectivity: High node density in sensor networks precludes them from being completely isolated from each other. Therefore, sensor nodes are expected to be highly connected. This, however, may not prevent the network topology from being variable and the network size from being shrinking due to sensor node failures. In addition, connectivity depends on the, possibly random, distribution of nodes.

- Coverage: In WSNs, each sensor node obtains a certain view of the environment. A given sensor's view of the environment is limited both in range and in accuracy; it can only cover a limited physical area of the environment. Hence, area coverage is also an important design parameter in WSNs.

- Data Aggregation: Since sensor nodes may generate significant redundant data, similar packets from multiple nodes can be aggregated so that the number of transmissions is reduced. Data aggregation is the combination of data from different sources according to a certain aggregation function, e.g., duplicate suppression, minima, maxima and average. This technique has been used to achieve energy efficiency and data transfer optimization in a number of routing protocols. Signal processing methods can also be used for data aggregation. In this case, it is referred to as data fusion where a node is capable of producing a more accurate output signal by using some techniques such as beamforming to combine the incoming signals and reducing the noise in these signals.

- Quality of Service: In some applications, data should be delivered within a certain period of time from the moment it is sensed, otherwise the data will be useless. Therefore bounded latency for data delivery is another condition for time-constrained applications. However, in many applications, conservation of energy, which is directly related to network lifetime, is considered relatively more important than the quality of data sent. As the energy gets depleted, the network may be required to reduce the quality of the results in order to reduce the energy dissipation in the nodes and hence lengthen the total network lifetime. Hence, energy-aware routing protocols are required to capture this requirement.

\section{Routing Protocols in WSNs}

In this section, we survey the state-of-the-art routing protocols for WSNs. In general, routing in WSNs can be divided into flat-based routing, hierarchical-based routing, and location-based routing depending on 
the network structure. In flat-based routing, all nodes are typically assigned equal roles or functionality. In hierarchical-based routing, however, nodes will play different roles in the network. In location-based routing, sensor nodes' positions are exploited to route data in the network. A routing protocol is considered adaptive if certain system parameters can be controlled in order to adapt to the current network conditions and available energy levels. Furthermore, these protocols can be classified into multipath-based, query-based, negotiation-based, QoS-based, or coherent-based routing techniques depending on the protocol operation. In addition to the above, routing protocols can be classified into three categories, namely, proactive, reactive, and hybrid protocols depending on how the source finds a route to the destination. In proactive protocols, all routes are computed before they are really needed, while in reactive protocols, routes are computed on demand. Hybrid protocols use a combination of these two ideas. When sensor nodes are static, it is preferable to have table driven routing protocols rather than using reactive protocols. A significant amount of energy is used in route discovery and setup of reactive protocols. Another class of routing protocols is called the cooperative routing protocols. In cooperative routing, nodes send data to a central node where data can be aggregated and may be subject to further processing, hence reducing route cost in terms of energy use. Many other protocols rely on timing and position information. We also shed some light on these types of protocols in this paper. In order to streamline this survey, we use a classification according to the network structure and protocol operation (routing criteria). The classification is shown in Figure 2 where numbers in the figure indicate the references.

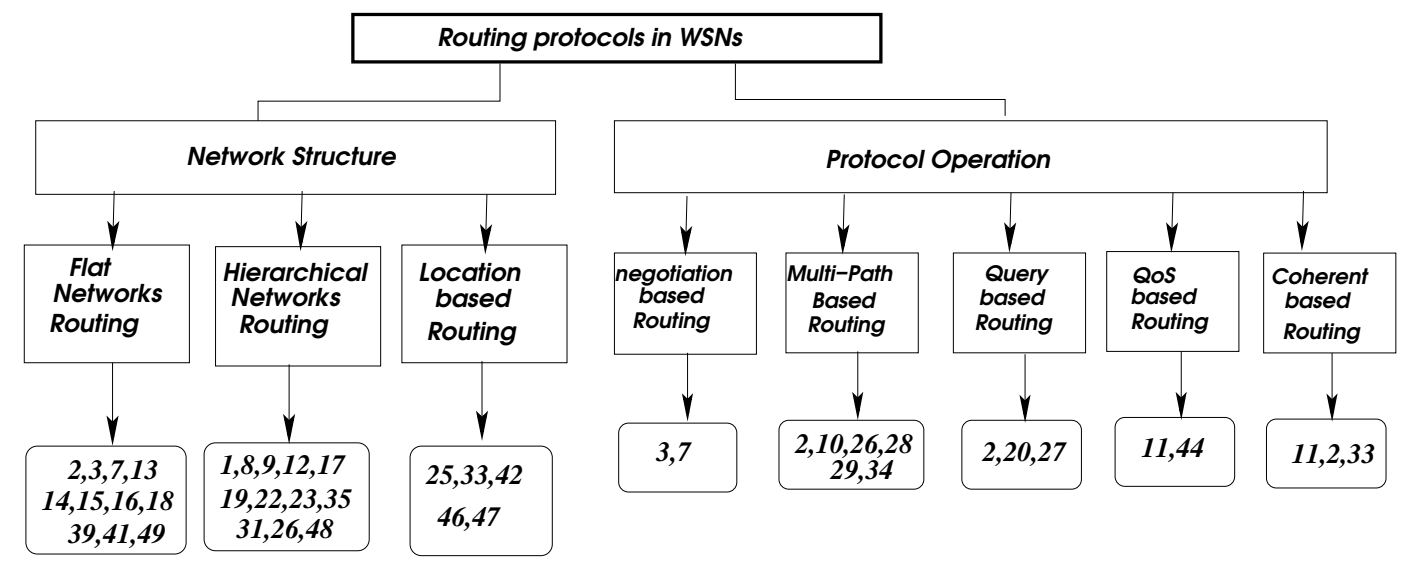

Figure 2: Routing protocols in WSNs: A taxonomy

In the rest of this section, we present a detailed overview of the main routing paradigms in WSNs. We start with network structure based protocols.

\subsection{Network Structure Based Protocols}

The underlying network structure can play significant role in the operation of the routing protocol in WSNs. In this section, we survey in details most of the protocols that fall below this category.

\subsubsection{Flat Routing}

The first category of routing protocols are the multihop flat routing protocols. In flat networks, each node typically plays the same role and sensor nodes collaborate together to perform the sensing task. Due to the large number of such nodes, it is not feasible to assign a global identifier to each node. This consideration 
has led to data centric routing, where the BS sends queries to certain regions and waits for data from the sensors located in the selected regions. Since data is being requested through queries, attribute-based naming is necessary to specify the properties of data. Early works on data centric routing, e.g., SPIN and directed diffusion [18] were shown to save energy through data negotiation and elimination of redundant data. These two protocols motivated the design of many other protocols which follow a similar concept. In the rest of this subsection, we summarize these protocols and highlight their advantages and their performance issues.

- Sensor Protocols for Information via Negotiation (SPIN): Heinzelman et.al. in [3] and [7] proposed a family of adaptive protocols called Sensor Protocols for Information via Negotiation (SPIN) that disseminate all the information at each node to every node in the network assuming that all nodes in the network are potential base-stations. This enables a user to query any node and get the required information immediately. These protocols make use of the property that nodes in close proximity have similar data, and hence there is a need to only distribute the data that other nodes do not posses. The SPIN family of protocols uses data negotiation and resource-adaptive algorithms. Nodes running SPIN assign a high-level name to completely describe their collected data (called meta-data) and perform meta-data negotiations before any data is transmitted. This assures that there is no redundant data sent throughout the network. The semantics of of the meta-data format is application-specific and is not specified in SPIN. For example, sensors might use their unique IDs to report meta-data if they cover a certain known region. In addition, SPIN has access to the current energy level of the node and adapts the protocol it is running based on how much energy is remaining. These protocols work in a time-driven fashion and distribute the information all over the network, even when a user does not request any data.

The SPIN family is designed to address the deficiencies of classic flooding by negotiation and resource adaptation. The SPIN family of protocols is designed based on two basic ideas:

1. Sensor nodes operate more efficiently and conserve energy by sending data that describe the sensor data instead of sending all the data; for example, image and sensor nodes must monitor the changes in their energy resources.

2. Conventional protocols like flooding or gossiping based routing protocols [6] waste energy and bandwidth when sending extra and un-necessary copies of data by sensors covering overlapping areas. The drawbacks of flooding include implosion, which is caused by duplicate messages sent to the same node, overlap when two nodes sensing the same region will send similar packets to the same neighbor, and resource blindness by consuming large amounts of energy without consideration for the energy constraints. Gossiping avoids the problem of implosion by just selecting a random node to send the packet to rather than broadcasting the packet blindly. However, this causes delays in propagation of data through the nodes.

SPIN's meta-data negotiation solves the classic problems of flooding, and thus achieving a lot of energy efficiency. SPIN is a 3-stage protocol as sensor nodes use three types of messages ADV, REQ and DATA to communicate. ADV is used to advertise new data, REQ to request data, and DATA is the actual message itself. The protocol starts when a SPIN node obtains new data that it is willing to share. It does so by broadcasting an ADV message containing meta-data. If a neighbor is interested 
in the data, it sends a REQ message for the DATA and the DATA is sent to this neighbor node. The neighbor sensor node then repeats this process with its neighbors. As a result, the entire sensor area will receive a copy of the data.

The SPIN family of protocols includes many protocols. The main two protocols are called SPIN-1 and SPIN-2, which incorporate negotiation before transmitting data in order to ensure that only useful information will be transferred. Also, each node has its own resource manager which keeps track of resource consumption, and is polled by the nodes before data transmission. The SPIN-1 protocol is a 3-stage protocol, as described above. An extension to SPIN-1 is SPIN-2, which incorporates threshold-based resource awareness mechanism in addition to negotiation. When energy in the nodes is abundant, SPIN-2 communicates using the 3-stage protocol of SPIN-1. However, when the energy in a node starts approaching a low energy threshold, it reduces its participation in the protocol, i.e., it participates only when it believes that it can complete all the other stages of the protocol without going below the low-energy threshold. In conclusion, SPIN-1 and SPIN-2 are simple protocols that efficiently disseminate data, while maintaining no per-neighbor state. These protocols are well-suited for an environment where the sensors are mobile because they base their forwarding decisions on local neighborhood information. Other protocols of the SPIN family are (please refer to [3] and [7] for more details):

- SPIN-BC: This protocol is designed for broadcast channels.

- SPIN-PP: This protocol is designed for a point to point communication, i.e., hop-by-hop routing.

- SPIN-EC: This protocol works similar to SPIN-PP, but with an energy heuristic added to it.

- SPIN-RL: When a channel is lossy, a protocol called SPIN-RL is used where adjustments are added to the SPIN-PP protocol to account for the lossy channel.

One of the advantages of SPIN is that topological changes are localized since each node needs to know only its single-hop neighbors. SPIN provides much energy savings than flooding and metadata negotiation almost halves the redundant data. However, SPINs data advertisement mechanism cannot guarantee the delivery of data. To see this, consider the application of intrusion detection where data should be reliably reported over periodic intervals and assume that nodes interested in the data are located far away from the source node and the nodes between source and destination nodes are not interested in that data, such data will not be delivered to the destination at all.

- Directed Diffusion: In [2], C. Intanagonwiwat et. al. proposed a popular data aggregation paradigm for WSNs, called directed diffusion. Directed diffusion is a data-centric (DC) and applicationaware paradigm in the sense that all data generated by sensor nodes is named by attribute-value pairs. The main idea of the DC paradigm is to combine the data coming from different sources enroute (in-network aggregation) by eliminating redundancy, minimizing the number of transmissions; thus saving network energy and prolonging its lifetime. Unlike traditional end-to-end routing, DC routing finds routes from multiple sources to a single destination that allows in-network consolidation of redundant data.

In directed diffusion, sensors measure events and create gradients of information in their respective neighborhoods. The base station requests data by broadcasting interests. Interest describes a task 
required to be done by the network. Interest diffuses through the network hop-by-hop, and is broadcast by each node to its neighbors. As the interest is propagated throughout the network, gradients are setup to draw data satisfying the query towards the requesting node, i.e., a BS may query for data by disseminating interests and intermediate nodes propagate these interests. Each sensor that receives the interest setup a gradient toward the sensor nodes from which it receives the interest. This process continues until gradients are setup from the sources back to the BS. More generally, a gradient specifies an attribute value and a direction. The strength of the gradient may be different towards different neighbors resulting in different amounts of information flow. At this stage, loops are not checked, but are removed at a later stage. Figure 3 shows an example of the working of directed diffusion ((a) sending interests, (b) building gradients, and (c) data dissemination). When interests fit gradients, paths of information flow are formed from multiple paths and then the best paths are reinforced so as to prevent further flooding according to a local rule. In order to reduce communication costs, data is aggregated on the way. The goal is to find a good aggregation tree which gets the data from source nodes to the BS. The BS periodically refreshes and re-sends the interest when it starts to receive data from the source(s). This is necessary because interests are not reliably transmitted throughout the network.

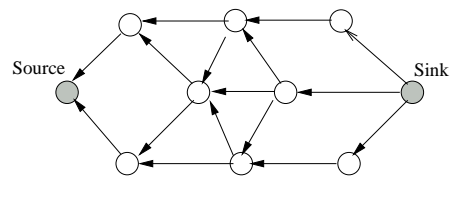

(a) Propagate Interest

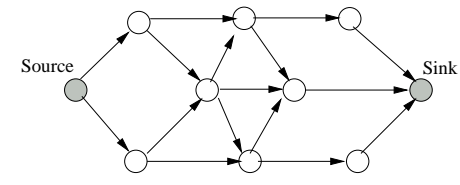

(b) Set up Gradients

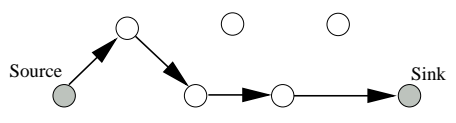

(c) Send data and path Reinforcement

Figure 3: An example of interest diffusion in sensor network

All sensor nodes in a directed diffusion-based network are application-aware, which enables diffusion to achieve energy savings by selecting empirically good paths and by caching and processing data in the network. Caching can increase the efficiency, robustness and scalability of coordination between sensor nodes which is the essence of the data diffusion paradigm. Other usage of directed diffusion is to spontaneously propagate an important event to some sections of the sensor network. Such type of information retrieval is well suited only for persistent queries where requesting nodes are not expecting data that satisfy a query for duration of time. This makes it unsuitable for one-time queries, as it is not worth setting up gradients for queries, which use the path only once.

The performance of data aggregation methods, used in the directed diffusion paradigm, are affected by a number of factors which includes the positions of the source nodes in the network, the number of sources, and the communication network topology. In order to investigate these factors, two models of source placement (shown in Figure 4), were studied in [2]. These models are called the event radius (ER) model, and the random sources (RS) model. In ER model, a single point in the network area is defined as the location of an event. This may correspond to a vehicle or some other 
phenomenon being tracked by the sensor nodes. All nodes within a distance $S$ (called the sensing range) of this event that are not BSs are considered to be data sources. The average number of sources is approximately $\pi S^{2} n$ in a unit area network with $n$ sensor nodes. In RS model, $k$ of the nodes that are not BSs are randomly selected to be sources. Unlike the ER model, the sources are not necessarily clustered near each other. In both models of source placement, and for a given energy budget, a greater number of sources can be connected to the BS. However, each one performs better in terms of energy consumption depending on the application. In conclusion, the energy savings with aggregation used in the directed diffusion can be transformed to provide a greater degree of robustness with respect to dynamics in the sensed phenomena.
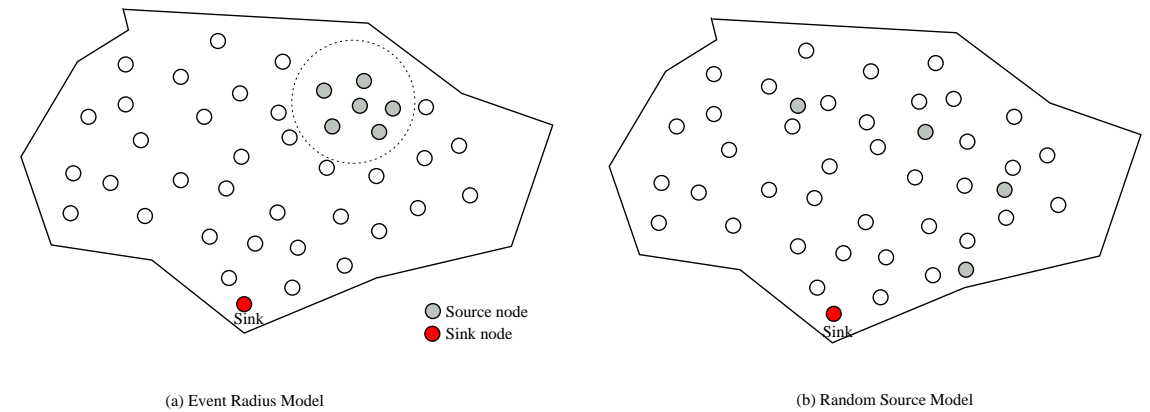

Figure 4: Two models used in data-centric routing paradigm, e.g., directed diffusion.

Directed diffusion differs from SPIN in two aspects. First, directed diffusion issues on demand data queries as the BS send queries to the sensor nodes by flooding some tasks. In SPIN, however, sensors advertise the availability of data allowing interested nodes to query that data. Second, all communication in directed diffusion is neighbor-to-neighbor with each node having the capability of performing data aggregation and caching. Unlike SPIN, there is no need to maintain global network topology in directed diffusion. However, directed diffusion may not be applied to applications (e.g., environmental monitoring) that require continuous data delivery to the BS. This is because the querydriven on demand data model may not help in this regard. Moreover, matching data to queries might require some extra overhead at the sensor nodes.

- Rumor routing: Rumor routing [14] is a variation of directed diffusion and is mainly intended for applications where geographic routing is not feasible. In general, directed diffusion uses flooding to inject the query to the entire network when there is no geographic criterion to diffuse tasks. However, in some cases there is only a little amount of data requested from the nodes and thus the use of flooding is unnecessary. An alternative approach is to flood the events if the number of events is small and the number of queries is large. The key idea is to route the queries to the nodes that have observed a particular event rather than flooding the entire network to retrieve information about the occurring events. In order to flood events through the network, the rumor routing algorithm employs long-lived packets, called agents. When a node detects an event, it adds such event to its local table, called events table, and generates an agent. Agents travel the network in order to propagate information about local events to distant nodes. When a node generates a query for an event, the nodes that know the route, may respond to the query by inspecting its event table. Hence, there is no need to flood the whole network, which reduces the communication cost. On the other hand, rumor routing 
maintains only one path between source and destination as opposed to directed diffusion where data can be routed through multiple paths at low rates. Simulation results showed that rumor routing can achieve significant energy savings when compared to event flooding and can also handle node's failure. However, rumor routing performs well only when the number of events is small. For a large number of events, the cost of maintaining agents and event-tables in each node becomes infeasible if there is not enough interest in these events from the BS. Moreover, the overhead associated with rumor routing is controlled by different parameters used in the algorithm such as time-to-live (TTL) pertaining to queries and agents. Since the nodes become aware of events through the event agents, the heuristic for defining the route of an event agent highly affects the performance of next hop selection in rumor routing.

- Minimum Cost Forwarding Algorithm (MCFA): The MCFA algorithm [18] exploits the fact that the direction of routing is always known, that is, towards the fixed external base-station. Hence, a sensor node need not have a unique ID nor maintain a routing table. Instead, each node maintains the least cost estimate from itself to the base-station. Each message to be forwarded by the sensor node is broadcast to its neighbors. When a node receives the message, it checks if it is on the least cost path between the source sensor node and the base-station. If this is the case, it re-broadcasts the message to its neighbors. This process repeats until the base-station is reached.

In MCFA, each node should know the least cost path estimate from itself to the base-station. This is obtained as follows. The base-station broadcasts a message with the cost set to zero while every node initially set its least cost to the base-station to infinity $(\infty)$. Each node, upon receiving the broadcast message originated at the base-station, checks to see if the estimate in the message plus the link on which it is received is less than the current estimate. If yes, the current estimate and the estimate in the broadcast message are updated. If the received broadcast message is updated, then it is re-sent; otherwise, it is purged and nothing further is done. However, the previous procedure may result in some nodes having multiple updates and those nodes far away from the base-station will get more updates from those closer to the base-station. To avoid this, the MCFA was modified to run a backoff algorithm at the setup phase. The backoff algorithm dictates that a node will not send the updated message until $a * l_{c}$ time units have elapsed from the time at which the message is updated, where $a$ is a constant and $l_{c}$ is the link cost from which the message was received.

- Gradient-Based Routing: Schurgers et al. [15] proposed another variant of directed diffusion, called Gradient-Based Routing (GBR). The key idea in GBR is to memorize the number of hops when the interest is diffused through the whole network. As such, each node can calculate a parameter called the height of the node, which is the minimum number of hops to reach the BS. The difference between a node's height and that of its neighbor is considered the gradient on that link. A packet is forwarded on a link with the largest gradient. GBR uses some auxiliary techniques such as data aggregation and traffic spreading in order to uniformly divide the traffic over the network. When multiple paths pass through a node, which acts as a relay node, that relay node may combine data according to a certain function. In GBR, three different data dissemination techniques have been discussed (1) Stochastic Scheme, where a node picks one gradient at random when there are two or more next hops that have the same gradient, (2) Energy-based scheme, where a node increases its height when its energy drops below a certain threshold, so that other sensors are discouraged 
from sending data to that node, and (3) Stream-based scheme, where new streams are not routed through nodes that are currently part of the path of other streams. The main objective of these schemes is to obtain a balanced distribution of the traffic in the network, thus increasing the network lifetime. Simulation results of GBR showed that GBR outperforms directed diffusion in terms of total communication energy.

\section{- Information-driven sensor querying (IDSQ) and Constrained anisotropic diffusion rout-} ing (CADR:) Two routing techniques, namely, information-driven sensor querying (IDSQ) and constrained anisotropic diffusion routing (CADR) were proposed in [16]. CADR aims to be a general form of directed diffusion. The key idea is to query sensors and route data in the network such that the information gain is maximized while latency and bandwidth are minimized. CADR diffuses queries by using a set of information criteria to select which sensors can get the data. This is achieved by activating only the sensors that are close to a particular event and dynamically adjusting data routes. The main difference from directed diffusion is the consideration of information gain in addition to the communication cost. In CADR, each node evaluates an information/cost objective and routes data based on the local information/cost gradient and end-user requirements. Estimation theory was used to model information utility measure. In IDSQ, the querying node can determine which node can provide the most useful information with the additional advantage of balancing the energy cost. However, IDSQ does not specifically define how the query and the information are routed between sensors and the BS. Therefore, IDSQ can be seen as a complementary optimization procedure. Simulation results showed that these approaches are more energy-efficient than directed diffusion where queries are diffused in an isotropic fashion and reaching nearest neighbors first.

- COUGAR: Another data-centric protocol called COUGAR [13] views the network as a huge distributed database system. The key idea is to use declarative queries in order to abstract query processing from the network layer functions such as selection of relevant sensors and so on. COUGAR utilizes in-network data aggregation to obtain more energy savings. The abstraction is supported through an additional query layer that lies between the network and application layers. COUGAR incorporates an architecture for the sensor database system where sensor nodes select a leader node to perform aggregation and transmit the data to the BS. The BS is responsible for generating a query plan, which specifies the necessary information about the data flow and in-network computation for the incoming query and send it to the relevant nodes. The query plan also describes how to select a leader for the query. The architecture provides in-network computation ability that can provide energy efficiency in situations when the generated data is huge. COUGAR provided a network-layer independent methods for data query. However, COUGAR has some drawbacks. First, the addition of query layer on each sensor node may add an extra overhead in terms of energy consumption and memory storage. Second, to obtain successful in-network data computation, synchronization among nodes is required (not all data are received at the same time from incoming sources) before sending the data to the leader node. Third, the leader nodes should be dynamically maintained to prevent them from being hot-spots (failure prone).

- ACQUIRE: In [41], Sadagopan et al. proposed a technique for querying sensor networks called ACtive QUery forwarding In sensoR nEtworks (ACQUIRE). Similar to COUGAR, ACQUIRE views the network as a distributed database where complex queries can be further divided into several 
sub queries. The operation of ACQUIRE can be described as follows. The BS node sends a query, which is then forwarded by each node receiving the query. During this, each node tries to respond to the query partially by using its pre-cached information and then forward it to another sensor node. If the pre-cached information is not up-to-date, the nodes gather information from their neighbors within a look-ahead of $d$ hops. Once the query is being resolved completely, it is sent back through either the reverse or shortest-path to the BS. Hence, ACQUIRE can deal with complex queries by allowing many nodes to send responses. Note that directed diffusion may not be used for complex queries due to energy considerations as directed diffusion also uses flooding-based query mechanism for continuous and aggregate queries. On the other hand, ACQUIRE can provide efficient querying by adjusting the value of the look-ahead parameter $d$. When $d$ is equal to network diameter, ACQUIRE mechanism behaves similar to flooding. However, the query has to travel more hops if $d$ is too small. A mathematical modeling was used to find an optimal value of the parameter $d$ for a grid of sensors where each node has 4 immediate neighbors. However, there is no validation of results through simulation. To select the next node for forwarding the query, ACQUIRE either picks it randomly or the selection is based on maximum potential of query satisfaction. Recall that selection of next node is based on either information gain (CADR and IDSQ) or query is forwarded to a node, which knows the path to the searched event (rumor routing).

- Energy Aware Routing: The objective of energy-aware routing protocol [39], a destination initiated reactive protocol, is to increase the network lifetime. Although this protocol is similar to directed diffusion, it differs in the sense that it maintains a set of paths instead of maintaining or enforcing one optimal path at higher rates. These paths are maintained and chosen by means of a certain probability. The value of this probability depends on how low the energy consumption of each path can be achieved. By having paths chosen at different times, the energy of any single path will not deplete quickly. This can achieve longer network lifetime as energy is dissipated more equally among all nodes. Network survivability is the main metric of this protocol. The protocol assumes that each node is addressable through a class-based addressing which includes the location and types of the nodes. The protocol initiates a connection through localized flooding, which is used to discover all routes between source/destination pair and their costs; thus building up the routing tables. The high-cost paths are discarded and a forwarding table is built by choosing neighboring nodes in a manner that is proportional to their cost. Then, forwarding tables are used to send data to the destination with a probability that is inversely proportional to the node cost. Localized flooding is performed by the destination node to keep the paths alive. When compared to directed diffusion, this protocol provides an overall improvement of $21.5 \%$ energy saving and a $44 \%$ increase in network lifetime. However, the approach requires gathering the location information and setting up the addressing mechanism for the nodes, which complicate route setup compared to the directed diffusion.

- Routing Protocols with Random Walks: The objective of random walks based routing technique [49] is to achieve load balancing in a statistical sense and by making use of multi-path routing in WSNs. This technique considers only large scale networks where nodes have very limited mobility. In this protocol, it is assumed that sensor nodes can be turned on or off at random times. Further, each node has a unique identifier but no location information is needed. Nodes were arranged such 
that each node falls exactly on one crossing point of a regular grid on a plane, but the topology can be irregular. To find a route from a source to its destination, the location information or lattice coordination is obtained by computing distances between nodes using the distributed asynchronous version of the well-known Bellman-Ford algorithm. An intermediate node would select as the next hop the neighboring node that is closer to the destination according to a computed probability. By carefully manipulating this probability, some kind of load balancing can be obtained in the network. The routing algorithm is simple as nodes are required to maintain little state information. Moreover, different routes are chosen at different times even for the same pair of source and destination nodes. However, the main concern about this protocol is that the topology of the network may not be practical.

\subsubsection{Hierarchical Routing}

Hierarchical or cluster-based routing, originally proposed in wireline networks, are well-known techniques with special advantages related to scalability and efficient communication. As such, the concept of hierarchical routing is also utilized to perform energy-efficient routing in WSNs. In a hierarchical architecture, higher energy nodes can be used to process and send the information while low energy nodes can be used to perform the sensing in the proximity of the target. This means that creation of clusters and assigning special tasks to cluster heads can greatly contribute to overall system scalability, lifetime, and energy efficiency. Hierarchical routing is an efficient way to lower energy consumption within a cluster and by performing data aggregation and fusion in order to decrease the number of transmitted messages to the BS. Hierarchical routing is mainly two-layer routing where one layer is used to select clusterheads and the other layer is used for routing. However, most techniques in this category are not about routing, rather on "who and when to send or process/aggregate" the information, channel allocation etc., which can be orthogonal to the multihop routing function.

- LEACH protocol: Heinzelman, et. al. [1] introduced a hierarchical clustering algorithm for sensor networks, called Low Energy Adaptive Clustering Hierarchy (LEACH). LEACH is a cluster-based protocol, which includes distributed cluster formation. LEACH randomly selects a few sensor nodes as clusterheads $(\mathrm{CHs})$ and rotate this role to evenly distribute the energy load among the sensors in the network. In $\mathrm{LEACH}$, the clusterhead $(\mathrm{CH})$ nodes compress data arriving from nodes that belong to the respective cluster, and send an aggregated packet to the base station in order to reduce the amount of information that must be transmitted to the base station. LEACH uses a TDMA/CDMA MAC to reduce inter-cluster and intra-cluster collisions. However, data collection is centralized and is performed periodically. Therefore, this protocol is most appropriate when there is a need for constant monitoring by the sensor network. A user may not need all the data immediately. Hence, periodic data transmissions are unnecessary which may drain the limited energy of the sensor nodes. After a given interval of time, a randomized rotation of the role of the $\mathrm{CH}$ is conducted so that uniform energy dissipation in the sensor network is obtained. The authors found, based on their simulation model, that only $5 \%$ of the nodes need to act as cluster heads.

The operation of LEACH is separated into two phases, the setup phase and the steady state phase. In the setup phase, the clusters are organized and $\mathrm{CH}$ are selected. In the steady state phase, the actual data transfer to the base station takes place. The duration of the steady state phase is 
longer than the duration of the setup phase in order to minimize overhead. During the setup phase, a predetermined fraction of nodes, $p$, elect themselves as $\mathrm{CHs}$ as follows. A sensor node chooses a random number, $r$, between 0 and 1 . If this random number is less than a threshold value, $T(n)$, the node becomes a cluster-head for the current round. The threshold value is calculated based on an equation that incorporates the desired percentage to become a cluster-head, the current round, and the set of nodes that have not been selected as a cluster-head in the last $(1 / \mathrm{P})$ rounds, denoted by $G$. It is given by:

$$
T(n)=\frac{p}{1-p(r \bmod (1 / p))} \text { if } n \in G
$$

where $G$ is the set of nodes that are involved in the $\mathrm{CH}$ election. Each elected $\mathrm{CH}$ broadcast an advertisement message to the rest of the nodes in the network that they are the new cluster-heads. All the non-cluster head nodes, after receiving this advertisement, decide on the cluster to which they want to belong to. This decision is based on the signal strength of the advertisement. The non cluster-head nodes inform the appropriate cluster-heads that they will be a member of the cluster. After receiving all the messages from the nodes that would like to be included in the cluster and based on the number of nodes in the cluster, the cluster-head node creates a TDMA schedule and assigns each node a time slot when it can transmit. This schedule is broadcast to all the nodes in the cluster.

During the steady state phase, the sensor nodes can begin sensing and transmitting data to the cluster-heads. The cluster-head node, after receiving all the data, aggregates it before sending it to the base-station. After a certain time, which is determined a priori, the network goes back into the setup phase again and enters another round of selecting new $\mathrm{CH}$. Each cluster communicates using different CDMA codes to reduce interference from nodes belonging to other clusters.

Although LEACH is able to increase the network lifetime, there are still a number of issues about the assumptions used in this protocol. LEACH assumes that all nodes can transmit with enough power to reach the BS if needed and that each node has computational power to support different MAC protocols. Therefore, it is not applicable to networks deployed in large regions. It also assumes that nodes always have data to send, and nodes located close to each other have correlated data. It is not obvious how the number of the predetermined $\mathrm{CHs}(p)$ is going to be uniformly distributed through the network. Therefore, there is the possibility that the elected CHs will be concentrated in one part of the network. Hence, some nodes will not have any $\mathrm{CHs}$ in their vicinity. Furthermore, the idea of dynamic clustering brings extra overhead, e.g. head changes, advertisements etc., which may diminish the gain in energy consumption. Finally, the protocol assumes that all nodes begin with the same amount of energy capacity in each election round, assuming that being a $\mathrm{CH}$ consumes approximately the same amount of energy for each node. The protocol should be extended to account for non-uniform energy nodes, i.e., use energy-based threshold. An extension to LEACH, LEACH with negotiation, was proposed in [1]. The main theme of the proposed extension is to precede data transfers with high-level negotiation using meta-data descriptors as in the SPIN protocol discussed in the previous section. This ensures that only data that provides new information is transmitted to the cluster-heads before being transmitted to the base station. Table 3.1.2 compares SPIN, LEACH, and the Directed Diffusion routing techniques according to different parameters. It is noted from the table that Directed Diffusion shows a promising approach for energy-efficient routing in WSNs due 
Table 1: Comparison between SPIN, LEACH and Directed Diffusion.

\begin{tabular}{|c|c|c|c|}
\hline & SPIN & LEACH & Directed Diffusion \\
\hline \hline Optimal Route & No & No & Yes \\
\hline Network Lifetime & Good & Very Good & Good \\
\hline Resource Awareness & Yes & Yes & Yes \\
\hline Use of Meta-Data & Yes & No & Yes \\
\hline
\end{tabular}

to the use of in-network processing.

- Power-Efficient Gathering in Sensor Information Systems (PEGASIS): In [17], an enhancement over LEACH protocol was proposed. The protocol, called Power-Efficient Gathering in Sensor Information Systems (PEGASIS), is a near optimal chain-based protocol. The basic idea of the protocol is that in order to extend network lifetime, nodes need only communicate with their closest neighbors and they take turns in communicating with the base-station. When the round of all nodes communicating with the base-station ends, a new round will start and so on. This reduces the power required to transmit data per round as the power draining is spread uniformly over all nodes. Hence, PEGASIS has two main objectives. First, increase the lifetime of each node by using collaborative techniques and as a result the network lifetime will be increased. Second, allow only local coordination between nodes that are close together so that the bandwidth consumed in communication is reduced. Unlike LEACH, PEGASIS avoids cluster formation and uses only one node in a chain to transmit to the BS instead of using multiple nodes.

To locate the closest neighbor node in PEGASIS, each node uses the signal strength to measure the distance to all neighboring nodes and then adjust the signal strength so that only one node can be heard. The chain in PEGASIS will consist of those nodes that are closest to each other and form a path to the base-station. The aggregated form of the data will be sent to the base-station by any node in the chain and the nodes in the chain will take turns in sending to the base-station. The chain construction is performed in a greedy fashion. Simulation results showed that PEGASIS is able to increase the lifetime of the network twice as much the lifetime of the network under the LEACH protocol. Such performance gain is achieved through the elimination of the overhead caused by dynamic cluster formation in LEACH and through decreasing the number of transmissions and reception by using data aggregation. Although the clustering overhead is avoided, PEGASIS still requires dynamic topology adjustment since a sensor node needs to know about energy status of its neighbors in order to know where to route its data. Such topology adjustment can introduce significant overhead especially for highly utilized networks. Moreover, PEGASIS assumes that each sensor node can be able to communicate with the BS directly. In practical cases, sensor nodes use multihop communication to reach the base-station. Also, PEGASIS assumes that all nodes maintain a complete database about the location of all other nodes in the network. The method of which the node locations are obtained is not outlined. In addition, PEGASIS assumes that all sensor nodes have the same level of energy and they are likely to die at the same time. Note also that PEGASIS introduces excessive delay for distant node on the chain. In addition, the single leader can become a bottleneck. Finally, although in most scenarios, sensors will be fixed or immobile as assumed in 
PEGASIS, some sensors may be allowed to move and hence affect the protocol functionality.

An extension to PEGASIS, called Hierarchical-PEGASIS was introduced in [21] with the objective of decreasing the delay incurred for packets during transmission to the BS. For this purpose, simultaneous transmissions of data are studied in order to avoid collisions through approaches that incorporates signal coding and spatial transmissions. In the later, only spatially separated nodes are allowed to transmit at the same time. The chain-based protocol with CDMA capable nodes, constructs a chain of nodes, that forms a tree like hierarchy, and each selected node in a particular level transmits data to the node in the upper level of the hierarchy. This method ensures data transmitting in parallel and reduces the delay significantly. Such hierarchical extension has been shown to perform better than the regular PEGASIS scheme by a factor of about 60 .

\section{- Threshold-sensitive Energy Efficient Protocols (TEEN and APTEEN):}

Two hierarchical routing protocols called TEEN (Threshold-sensitive Energy Efficient sensor Network protocol), and APTEEN (Adaptive Periodic Threshold-sensitive Energy Efficient sensor Network protocol) are proposed in [8] and [9], respectively. These protocols were proposed for time-critical applications. In TEEN, sensor nodes sense the medium continuously, but the data transmission is done less frequently. A cluster head sensor sends its members a hard threshold, which is the threshold value of the sensed attribute and a soft threshold, which is a small change in the value of the sensed attribute that triggers the node to switch on its transmitter and transmit. Thus the hard threshold tries to reduce the number of transmissions by allowing the nodes to transmit only when the sensed attribute is in the range of interest. The soft threshold further reduces the number of transmissions that might have otherwise occurred when there is little or no change in the sensed attribute. A smaller value of the soft threshold gives a more accurate picture of the network, at the expense of increased energy consumption. Thus, the user can control the trade-off between energy efficiency and data accuracy. When cluster-heads are to change (see Figure 5(a)), new values for the above parameters are broadcast. The main drawback of this scheme is that, if the thresholds are not received, the nodes will never communicate, and the user will not get any data from the network at all.

The nodes sense their environment continuously. The first time a parameter from the attribute set reaches its hard threshold value, the node switches its transmitter on and sends the sensed data. The sensed value is stored in an internal variable, called Sensed Value (SV). The nodes will transmit data in the current cluster period only when the following conditions are true: (1) The current value of the sensed attribute is greater than the hard threshold (2) The current value of the sensed attribute differs from SV by an amount equal to or greater than the soft threshold.

Important features of TEEN include its suitability for time critical sensing applications. Also, since message transmission consumes more energy than data sensing, so the energy consumption in this scheme is less than the proactive networks. The soft threshold can be varied. At every cluster change time, a fresh parameters are broadcast and so, the user can change them as required.

APTEEN, on the other hand, is a hybrid protocol that changes the periodicity or threshold values used in the TEEN protocol according to the user needs and the type of the application. In APTEEN, the cluster-heads broadcasts the following parameters (see Figure 5(b)): 


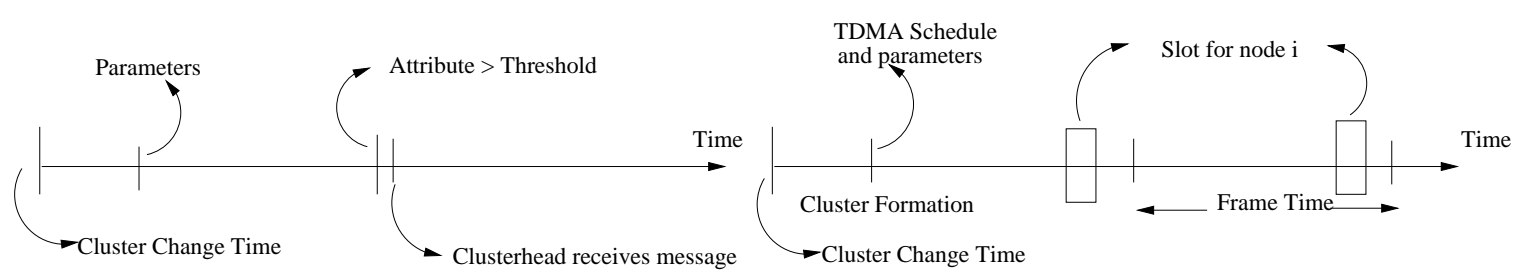

(a) operation of TEEN

(b) operation of APTEEN

Figure 5: Time line for the operation of (a) TEEN and (b) APTEEN

1. Attributes (A): this is a set of physical parameters which the user is interested in obtaining information about.

2. Thresholds: this parameter consists of the Hard Threshold (HT) and the Soft Threshold (ST).

3. Schedule: this is a TDMA schedule, assigning a slot to each node.

4. Count Time (CT): it is the maximum time period between two successive reports sent by a node.

The node senses the environment continuously, and only those nodes which sense a data value at or beyond the hard threshold transmit. Once a node senses a value beyond HT, it transmits data only when the value of that attribute changes by an amount equal to or greater than the ST. If a node does not send data for a time period equal to the count time, it is forced to sense and retransmit the data. A TDMA schedule is used and each node in the cluster is assigned a transmission slot. Hence, APTEEN uses a modified TDMA schedule to implement the hybrid network. The main features of the APTEEN scheme include the following. It combines both proactive and reactive policies. It offers a lot of flexibility by allowing the user to set the count-time interval (CT), and the threshold values for the energy consumption can be controlled by changing the count time as well as the threshold values. The main drawback of the scheme is the additional complexity required to implement the threshold functions and the count time. Simulation of TEEN and APTEEN has shown that these two protocols outperform LEACH. The experiments have demonstrated that APTEENs performance is somewhere between LEACH and TEEN in terms of energy dissipation and network lifetime. TEEN gives the best performance since it decreases the number of transmissions. The main drawbacks of the two approaches are the overhead and complexity associated with forming clusters at multiple levels, the method of implementing threshold-based functions, and how to deal with attribute-based naming of queries.

- Small Minimum Energy Communication Network (MECN): In [22], a protocol is proposed that computes an energy-efficient subnetwork, namely the minimum energy communication network (MECN) for a certain sensor network by utilizing low power GPS. MECN identifies a relay region for every node. The relay region consists of nodes in a surrounding area where transmitting through those nodes is more energy efficient than direct transmission. The relay region for node pair (i, r) is depicted in Fig. 10, redrawn from [39]. The enclosure of a node $\mathrm{i}$ is then created by taking the union of all relay regions that node i can reach. The main idea of MECN is to find a sub-network, which will have less number of nodes and require less power for transmission between any two particular nodes. In this way, global minimum power paths are found without considering all the nodes in the network. 
This is performed using a localized search for each node considering its relay region. MECN is selfreconfiguring and thus can dynamically adapt to nodes failure or the deployment of new sensors. The small minimum energy communication network (SMECN) [23] is an extension to MECN. In MECN, it is assumed that every node can transmit to every other node, which is not possible every time. In SMECN possible obstacles between any pair of nodes are considered. However, the network is still assumed to be fully connected as in the case of MECN. The subnetwork constructed by SMECN for minimum energy relaying is provably smaller (in terms of number of edges) than the one constructed in MECN. Hence, the subnetwork (i.e., subgraph $G^{\prime}$ ) constructed by SMECN is smaller than the one constructed by MECN if the broadcast region is circular around the broadcasting node for a given power setting. Subgraph $G^{\prime}$ of graph $G$, which represents the sensor network, minimizes the energy usage satisfying the following conditions: (1)the number of edges in $G^{\prime}$ is less than in $G$ while containing all nodes in $G,(2)$ the energy required to transmit data from a node to all its neighbors in subgraph $G^{\prime}$ is less than the energy required to transmit to all its neighbors in graph $G$. Assume that $r=\left(u, u_{1}, \ldots, u_{k-1}, v\right)$ is a path between $u$ and $v$ that spans $k-1$ intermediate nodes $u_{1}, \ldots, u_{k-1}$. The total power consumption of one path like $r$ is given by:

$$
C(r)=\sum_{i=0}^{k-1}\left(p\left(u_{i}, u_{i+1}\right)+c\right)
$$

where $u=u_{0}$ and $v=u_{k}$ and the power required to transmit data under this protocol is

$$
p(u, v)=t . d(u, v)^{n}
$$

for some appropriate constant $t, n$ is the path-loss exponent of outdoor radio propagation models $n \geq 2$, and $d(u, v)$ is the distance between $u$ and $v$. It is assumed that a reception at the receiver takes a constant amount of power denoted by $c$. The subnetwork computed by SMECN helps sending messages on minimum-energy paths. However, the proposed algorithm is local in the sense that it does not actually find the minimum-energy path, it just constructs a subnetwork in which it is guaranteed to exist. Moreover, the subnetwork constructed by SMECN makes it more likely that the path used is one that requires less energy consumption. In addition, finding a sub-network with smaller number of edges introduces more overhead in the algorithm.

- Self Organizing Protocol (SOP): Subramanian et al. [12] describes a self-organizing protocol and an application taxonomy that was used to build architecture used to support heterogeneous sensors. Furthermore, these sensors can be mobile or stationary. Some sensors probe the environment and forward the data to a designated set of nodes that act as routers. Router nodes are stationary and form the backbone for communication. Collected data are forwarded through the routers to the more powerful BS nodes. Each sensing node should be able to reach a router in order to be part of the network. A routing architecture that requires addressing of each sensor node has been proposed. Sensing nodes are identifiable through the address of the router node they are connected to. The routing architecture is hierarchical where groups of nodes are formed and merge when needed. Local Markov Loops (LML) algorithm, which performs a random walk on spanning trees of a graph, was used to support fault tolerance and as a means of broadcasting. Such approach is similar to the idea of virtual grid used in some other protocols that will be discussed later under locationbased routing protocols. In this approach, sensor nodes can be addressed individually in the routing 
architecture, and hence it is suitable for applications where communication to a particular node is required. Furthermore, this algorithm incurs a small cost for maintaining routing tables and keeping a balanced routing hierarchy. It was also found that the energy consumed for broadcasting a message is less than that consumed in the SPIN protocol. This protocol, however, is not an on-demand protocols especially in the organization phase of algorithm. Therefore, introducing extra overhead. Another issue is related to the formation of hierarchy. It could happen that there are many cuts in the network, and hence the probability of applying reorganization phase increases, which will be an expensive operation.

- Sensor Aggregates Routing: In [35], a set of algorithms for constructing and maintaining sensor aggregates were proposed. The objective is to collectively monitor target activity in a certain environment (target tracking applications). A sensor aggregate comprises those nodes in a network that satisfy a grouping predicate for a collaborative processing task. The parameters of the predicate depend on the task and its resource requirements. The formation of appropriate sensor aggregates were discussed in [35] in terms of allocating resources to sensing and communication tasks. Sensors in a sensor field is divided into clusters according to their sensed signal strength, so that there is only one peak per cluster. Then, local cluster leaders are elected. One peak may represent one target, multiple targets, or no target in case the peak is generated by noise sources. To elect a leader, information exchanges between neighboring sensors are necessary. If a sensor, after exchanging packets with all its one-hop neighbors, finds that it is higher than all its one-hop neighbors on the signal field landscape, it declares itself a leader. This leader-based tracking algorithm assumes the unique leader knows the geographical region of the collaboration.

Three algorithms were proposed in [35]. First, a lightweight protocol, Distributed Aggregate Management (DAM), for forming sensor aggregates for a target monitoring task. The protocol comprises a decision predicate $P$ for each node to decide if it should participate in an aggregate and a message exchange scheme $M$ about how the grouping predicate is applied to nodes. A node determines if it belongs to an aggregate based on the result of applying the predicate to the data of the node as well as information from other nodes. Aggregates are formed when the process eventually converges. Second, Energy-Based Activity Monitoring (EBAM) algorithm estimate the energy level at each node by computing the signal impact area, combining a weighted form of the detected target energy at each impacted sensor assuming that each target sensor has equal or constant energy level. The third algorithm, Expectation-Maximization Like Activity Monitoring (EMLAM), removes the constant and equal target energy level assumption. EMLAM estimates the target positions and signal energy using received signals, and uses the resulting estimates to predict how signals from the targets may be mixed at each sensor. This process is iterated, until the estimate is sufficiently good.

The distributed track initiation management scheme, combined with the leader-based tracking algorithm described in [35], forms a scalable system. The system works well in tracking multiple targets when the targets are not interfering, and it can recover from inter-target interference once the targets move apart.

- Virtual Grid Architecture routing (VGA): An energy-efficient routing paradigm is proposed in [31] that utilizes data aggregation and in-network processing to maximize the network lifetime. Due to the node stationarity and extremely low mobility in many applications in WSNs, a reasonable 
approach is to arrange nodes in a fixed topology as was briefly mentioned in [25]. A GPS-free approach [21] is used to build clusters that are fixed, equal, adjacent, and non-overlapping with symmetric shapes. In [31], square clusters were used to obtain a fixed rectilinear virtual topology. Inside each zone, a node is optimally selected to act as clusterhead. Data aggregation is performed at two levels: local and then global. The set of clusterheads, also called Local Aggregators (LAs), perform the local aggregation, while a subset of these LAs are used to perform global aggregation. However, the determination of an optimal selection of global aggregation points, called Master Aggregators (MAs), is NP-hard problem. Figure 6 illustrates an example of fixed zoning and the resulting virtual grid architecture (VGA) used to perform two level data aggregation. Note that the location of the base station is not necessarily at the extreme corner of the grid, rather it can be located at any arbitrary place.

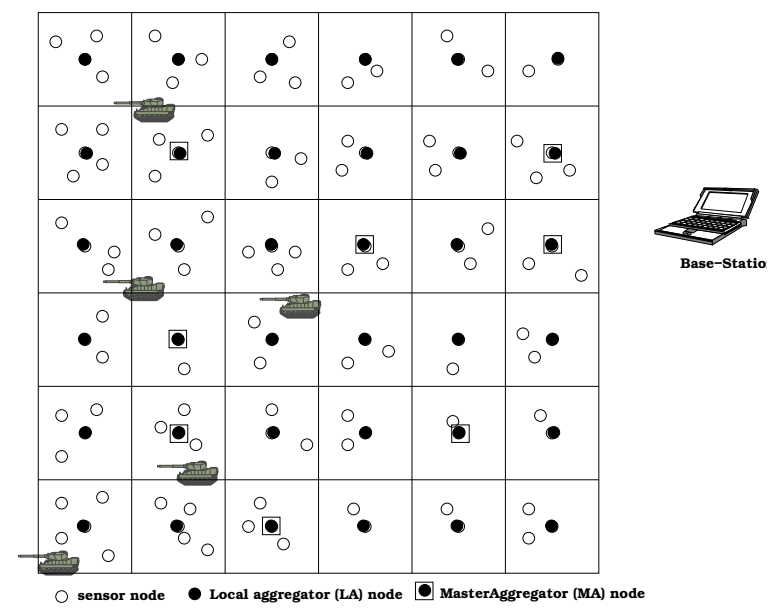

Figure 6: Regular shape tessellation applied to the network area. In each zone, a clusterhead is selected for local aggregation. A subset of those clusterheads, called Master nodes, are optimally selected to do global aggregation.

Two solution strategies for the routing with data aggregation problem are presented in [31]: an exact algorithm using an Integer Linear Program (ILP) formulation and several near optimal, but simple and efficient, approximate algorithms, namely, a genetics algorithms based heuristic, a k-means heuristic, and a greedy based heuristic. In [48], another efficient heuristic, called Clustering-Based Aggregation Heuristic (CBAH), was also proposed to minimize energy consumption in the network, and hence prolong the network lifetime. The objective of all algorithms is to select a number of MAs out of the LAs, that maximize the network lifetime. For a realistic scenario, it is assumed in [31] that LA nodes form, possibly overlapping, groups. Members of each group are sensing the same phenomenon, and hence their readings are correlated. However, each LA node that exists in the overlapping region, will be sending data to its associated MA for each of the groups it belongs to. It was noted in [48] that the problem of assigning MAs to LAs in CBAH is similar to the classical bin-packing problem, but with a major difference being that neither the identities nor the amount of power that each MA will be using for different LAs are known. In CBAH, the set of MAs are selected based on incremental filling of the some bins with capacities. Besides being fast and scalable to large sensor networks, the approximate algorithms in [31, 48] produce results which are not far from the optimal solution. 
- Hierarchical Power-aware Routing (HPAR): In [26], a hierarchical power-aware routing was proposed. The protocol divides the network into groups of sensors. Each group of sensors in geographic proximity are clustered together as a zone and each zone is treated as an entity. To perform routing, each zone is allowed to decide how it will route a message hierarchically across the other zones such that the battery lives of the nodes in the system are maximized. Message are routed along the path which has the maximum over all the minimum of the remaining power, called the max-min path. The motivation is that using nodes with high residual power may be expensive as compared to the path with the minimal power consumption. An approximation algorithm, called the max-min zPmin algorithm, was proposed in [26]. The crux of the algorithm is based on the tradeoff between minimizing the total power consumption and maximizing the minimal residual power of the network. Hence, the algorithm tries to enhance a max-min path by limiting its power consumption as follows. First, the algorithm finds the path with the least power consumption $\left(P_{\min }\right)$ by using the Dijkstra algorithm. Second, the algorithm finds a path that maximizes the minimal residual power in the network. The proposed algorithm tries to optimizes both solution criteria. This is achieved by relaxing the minimal power consumption for the message to be equal to $z P \min$ with parameter $z \geq 1$ to restrict the power consumption for sending one message to $z P \min$. The algorithm consumes at most $z P \min$ while maximizing the minimal residual power fraction.

Another algorithm, called zone-based routing, that relies on max-min zPmin is also proposed in [26]. Zone-base routing is a hierarchical approach where the area covered by the (sensor) network is divided into a small number of zones. To send a message across the entire area, a global path from zone to zone is found. The sensors in a zone autonomously direct local routing and participate in estimating the zone power level. Each message is routed across the zones using information about the zone power estimates. A global controller for message routing is assigned the role of managing the zones. This may be the node with the highest power. If the network can be divided into a relatively small number of zones, the scale for the global routing algorithm is reduced. The global information required to send each message across is summarized by the power level estimate of each zone. A zone graph was used to represent connected neighboring zone vertices if the current zone can go to the next neighboring zone in that direction. Each zone vertex has a power level of 1 . Each zone direction vertex is labelled by its estimated power level computed by a procedure, which is a modified Bellman-Ford algorithm. Moreover, two algorithms were outlined for local and global path selection using the zone graph.

- Two-Tier Data Dissemination (TTDD): An approach in [19], called Two-Tier Data Dissemination (TTDD), provides data delivery to multiple mobile bas-stations. In TTDD, each data source proactively builds a grid structure which is used to disseminate data to the mobile sinks by assuming that sensor nodes are stationary and location-aware. In TTDD, sensor nodes are stationary and location-aware, whereas sinks may change their locations dynamically. Once an event occurs, sensors surrounding it process the signal and one of them becomes the source to generate data reports. Sensor nodes are aware of their mission which will not change frequently. To build the grid structure, a data source chooses itself as the start crossing point of the grid, and sends a data announcement message to each of its four adjacent crossing points using simple greedy geographical forwarding. When the message reaches a node that is closest to the crossing point (specified in the message), it will stop. 
During this process, each intermediate node stores the source information and further forwards the message to its adjacent crossing points except the one from which the message comes from. This process continues until the message stops at the border of the network. The nodes that store the source information are chosen as dissemination points. After this process, the grid structure is obtained. Using the grid, a BS can flood a query, which will be forwarded to the nearest dissemination point in the local cell to receive data. Then the query is forwarded along other dissemination points upstream to the source. The requested data then flows down in the reverse path to the sink. Trajectory forwarding is employed as the BS moves in the sensor field. Although TTDD is an efficient routing approach, there are some concerns about how the algorithm obtains location information, which is required to set up the grid structure. The length of a forwarding path in TTDD is larger than the length of the shortest path. The authors of TTDD believe that the suboptimality in the path length is worth the gain in scalability. Finally, how would TTDD perform if mobile sensor nodes are allowed to move in the network is still an open question. Comparison results between TTDD and directed diffusion showed that TTDD can achieve longer lifetimes and data delivery delays. However, the overhead associated with maintaining and recalculating the grid as network topology changes may be high. Furthermore, TTDD assumed the availability of very accurate positioning system which is not yet available for WSNs.

The above mentioned flat and hierarchical protocols are different in many aspects. At this point, we compare the different routing approaches for flat and hierarchical sensor networks, which is shown in

Table 3.1.2.

Table 2: Hierarchical vs. flat topologies routing

\begin{tabular}{l|l}
\hline Hierarchical routing & Flat routing \\
\hline \hline Reservation-based scheduling & Contention-based scheduling \\
\hline Collisions avoided & Collision overhead present \\
\hline Reduced duty cycle due to periodic sleeping & Variable duty cycle by controlling sleep time of nodes \\
\hline Data aggregation by clusterhead & $\begin{array}{l}\text { node on multihop path aggregates incoming data from } \\
\text { neighbors }\end{array}$ \\
\hline Simple but non-optimal routing & $\begin{array}{l}\text { Routing can be made optimal but with an added com- } \\
\text { plexity. }\end{array}$ \\
\hline Requires global and local synchronization & Links formed on the fly without synchronization \\
\hline Overhead of cluster formation throughout the network & $\begin{array}{l}\text { Routes formed only in regions that have data for trans- } \\
\text { mission }\end{array}$ \\
\hline $\begin{array}{l}\text { Lower latency as multiple hops network formed by } \\
\text { clusterheads always available }\end{array}$ & $\begin{array}{l}\text { Latency in waking up intermediate nodes and setting } \\
\text { up the multipath }\end{array}$ \\
\hline Energy dissipation is uniform & Energy dissipation depends on traffic patterns \\
\hline Energy dissipation cannot be controlled & Energy dissipation adapts to traffic pattern \\
\hline \hline Fair channel allocation & Fairness not guaranteed \\
\hline \hline
\end{tabular}

\subsubsection{Location based routing protocols}

In this kind of routing, sensor nodes are addressed by means of their locations. The distance between neighboring nodes can be estimated on the basis of incoming signal strengths. Relative coordinates of neighboring nodes can be obtained by exchanging such information between neighbors [20], [21], [30]. 
Alternatively, the location of nodes may be available directly by communicating with a satellite, using GPS (Global Positioning System), if nodes are equipped with a small low power GPS receiver [25]. To save energy, some location based schemes demand that nodes should go to sleep if there is no activity. More energy savings can be obtained by having as many sleeping nodes in the network as possible. The problem of designing sleep period schedules for each node in a localized manner was addressed in [33, 25]. In the rest of this section, we review most of the location or geographic based routing protocols.

- Geographic Adaptive Fidelity (GAF): GAF [25] is an energy-aware location-based routing algorithm designed primarily for mobile ad hoc networks, but may be applicable to sensor networks as well. The network area is first divided into fixed zones and form a virtual grid. Inside each zone, nodes collaborate with each other to play different roles. For example, nodes will elect one sensor node to stay awake for a certain period of time and then they go to sleep. This node is responsible for monitoring and reporting data to the BS on behalf of the nodes in the zone. Hence, GAF conserves energy by turning off unnecessary nodes in the network without affecting the level of routing fidelity. Each node uses its GPS-indicated location to associate itself with a point in the virtual grid. Nodes associated with the same point on the grid are considered equivalent in terms of the cost of packet routing. Such equivalence is exploited in keeping some nodes located in a particular grid area in sleeping state in order to save energy. Thus, GAF can substantially increase the network lifetime as the number of nodes increases. There are three states defined in GAF. These states are discovery, for determining the neighbors in the grid, active reflecting participation in routing and sleep when the radio is turned off. In order to handle the mobility, each node in the grid estimates its leaving time of grid and sends this to its neighbors. The sleeping neighbors adjust their sleeping time accordingly in order to keep the routing fidelity. Before the leaving time of the active node expires, sleeping nodes wake up and one of them becomes active. GAF is implemented both for non-mobility (GAFbasic) and mobility (GAF-mobility adaptation) of nodes. Figure 7 shows an example of fixed zoning that can be used in sensor networks similar to the one proposed in [25]. The fixed clusters in [25]

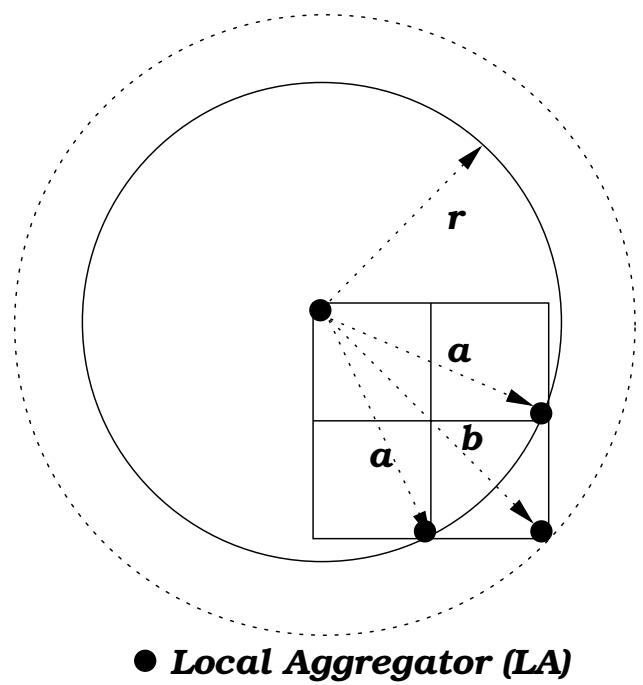

Figure 7: An example of zoning in sensor networks.

are selected to be equal and square. The selection of the square size is dependent on the required transmitting power and the communication direction. A vertical and horizontal communication is 
guaranteed to happen if the signal travels a distance of $a=\frac{r}{\sqrt{5}}$, chosen such that any two sensor nodes in adjacent vertical or horizontal clusters can communicate directly. For a diagonal communication to happen, the signal has to span a distance of $b=\frac{r}{2 \sqrt{2}}$. The issue is how to schedule roles for the nodes to act as clusterheads. A clusterhead can ask the sensor nodes in its cluster to switch on and start gathering data if it senses an object. Then, clusterhead is responsible for receiving raw data from other nodes in its cluster and forward it to the BS. The authors in [25] assumed that sensor nodes can know their locations using GPS cards, which is inconceivable with the current technology. GAF strives to keep the network connected by keeping a representative node always in active mode for each region on its virtual grid. Simulation results show that GAF performs at least as well as a normal ad hoc routing protocol in terms of latency and packet loss and increases the lifetime of the network by saving energy. Although GAF is a location-based protocol, it may also be considered as a hierarchical protocol, where the clusters are based on geographic location. For each particular grid area, a representative node acts as the leader to transmit the data to other nodes. The leader node however, does not do any aggregation or fusion as in the case of other hierarchical protocols discussed earlier in this article.

- Geographic and Energy Aware Routing (GEAR): Yu et al. [42] discussed the use of geographic information while disseminating queries to appropriate regions since data queries often include geographic attributes. The protocol, called Geographic and Energy Aware Routing (GEAR), uses energy aware and geographically-informed neighbor selection heuristics to route a packet towards the destination region. The key idea is to restrict the number of interests in directed diffusion by only considering a certain region rather than sending the interests to the whole network. By doing this, GEAR can conserve more energy than directed diffusion.

Each node in GEAR keeps an estimated cost and a learning cost of reaching the destination through its neighbors. The estimated cost is a combination of residual energy and distance to destination. The learned cost is a refinement of the estimated cost that accounts for routing around holes in the network. A hole occurs when a node does not have any closer neighbor to the target region than itself. If there are no holes, the estimated cost is equal to the learned cost. The learned cost is propagated one hop back every time a packet reaches the destination so that route setup for next packet will be adjusted. There are two phases in the algorithm: (1) Forwarding packets towards the target region: Upon receiving a packet, a node checks its neighbors to see if there is one neighbor, which is closer to the target region than itself. If there is more than one, the nearest neighbor to the target region is selected as the next hop. If they are all further than the node itself, this means there is a hole. In this case, one of the neighbors is picked to forward the packet based on the learning cost function. This choice can then be updated according to the convergence of the learned cost during the delivery of packets, and (2) Forwarding the packets within the region: If the packet has reached the region, it can be diffused in that region by either recursive geographic forwarding or restricted flooding. Restricted flooding is good when the sensors are not densely deployed. In high-density networks, recursive geographic flooding is more energy efficient than restricted flooding. In that case, the region is divided into four sub regions and four copies of the packet are created. This splitting and forwarding process continues until the regions with only one node are left.

In [42], GEAR was compared to a similar non-energy-aware routing protocol GPSR [43], which is one 
of the earlier works in geographic routing that uses planar graphs to solve the problem of holes. In case of GPSR, the packets follow the perimeter of the planar graph to find their route. Although the GPSR approach reduces the number of states a node should keep, it has been designed for general mobile ad hoc networks and requires a location service to map locations and node identifiers. GEAR not only reduces energy consumption for the route setup, but also performs better than GPSR in terms of packet delivery. The simulation results show that for an uneven traffic distribution, GEAR delivers $70 \%$ to $80 \%$ more packets than (GPSR). For uniform traffic pairs GEAR delivers 25\%-35\% more packets than GPSR.

- MFR, DIR, and GEDIR: Stojmenovic and Lin [46] described and discussed basic localized routing algorithms. These protocols deal with basic distance, progress, and direction based methods. The key issues are forward direction and backward direction. A source node or any intermediate node will select one of its neighbors according to a certain criterion. The routing methods, which belong to this category, are MFR (Most Forward within Radius), GEDIR (The Geographic Distance Routing) that is a variant of greedy algorithms, 2-hop greedy method, alternate greedy method and DIR (compass routing method). GEDIR algorithm is a greedy algorithm that always moves the packet to the neighbor of the current vertex whose distance to the destination is minimized. The algorithm fails when the packet crosses the same edge twice in succession. In most cases, the MFR and Greedy methods have the same path to destination. In the DIR method, the best neighbor has the closest direction (that is, angle) toward the destination. That is, the neighbor with the minimum angular distance from the imaginary line joining the current node and the destination is selected. In MFR method, the best neighbor $A$ will minimize the dot product $\overline{D A} \cdot \overline{D S}$, where $S, D$ are the source and destination nodes, respectively, and $\overline{S D}$ represents the Euclidian distance between the two nodes $S, D$. Alternatively, one can maximize the dot product $\overline{S D} \cdot \overline{S A}$. Each method stops forwarding the message at a node for which the best choice is to return the message back to a previous node. GEDIR and MFR methods are loop-free, while DIR method may create loops, unless past traffic is memorized or a time-stamp is enforced [46].

A comparison study [46] between these algorithms showed that the three basic algorithms had comparable performance in terms of delivery rate and average dilation. Moreover, simulations revealed that the nodes in MFR and Greedy methods select the same forwarding neighbor in more than $99 \%$ cases and the entire selected paths were identical in most of the cases.

- The Greedy Other Adaptive Face Routing (GOAFR): In [47], a geometric ad-hoc routing algorithm combining greedy and face routing was proposed. We will now briefly review the key points of GOAFR in this section. The greedy algorithm of GOAFR always picks the neighbor closest to a node to be next node for routing. However, it can be easily stuck at some local minimum, i.e. no neighbor is closer to a node than the current node. Other Face Routing (OFR) is a variant of Face Routing (FR). The Face Routing (FR) algorithm [46] is the first one that guarantees success if the source and the destination are connected. However, the worst-case cost of FR is proportional to the size of the network in terms of number of nodes. The first algorithm that can compete with the best route in the worst-case is the Adaptive Face Routing (AFR) algorithm. Moreover, by a lower bound argument, AFR is shown to be asymptotically worst-case optimal. But AFR is not average-case efficient. OFR utilizes the face structure of planar graphs such that the message is 
routed from node $s$ to node $t$ by traversing a series of face boundaries. The aim is to find the best node on the boundary, i.e., the closest node to the destination $t$ by using geometric planes. When finished, the algorithm returns to $s$ the best node on the boundary. The simple greedy algorithm behaves well in dense networks, but it fails for very simple configurations as was shown in [47]. It was shown that GOAFR algorithm can achieve both worst-case optimality and average-case efficiency. Based on the simulation results of GOAFR, there are several ways to further improve the averagecase performance. It was also shown that GOAFR outperforms other prominent algorithms, such as GPSR or AFR.

- SPAN: Another position based algorithm called SPAN [33] selects some nodes as coordinators based on their positions. The coordinators form a network backbone that is used to forward messages. A node should become a coordinator if two neighbors of a non-coordinator node cannot reach each other directly or via one or two coordinators (3 hop reachability). New and existing coordinators are not necessarily neighbors in [33], which, in effect, makes the design less energy efficient because of the need to maintain the positions of two or three hop neighbors in the complicated SPAN algorithm.

\subsection{Routing Protocols based on Protocol Operation}

In this section, we review routing protocols that different routing functionality. It should be noted that some of these protocols may fall below one or more of the above routing categories.

\subsubsection{Multipath routing protocols}

In this subsection, we study the routing protocols that use multiple paths rather than a single path in order to enhance the network performance. The fault tolerance (resilience) of a protocol is measured by the likelihood that an alternate path exists between a source and a destination when the primary path fails. This can be increased by maintaining multiple paths between the source and the destination at the expense of an increased energy consumption and traffic generation. These alternate paths are kept alive by sending periodic messages. Hence, network reliability can be increased at the expense of increased overhead of maintaining the alternate paths.

The authors in [28] proposed an algorithm which will route data through a path whose nodes have the largest residual energy. The path is changed whenever a better path is discovered. The primary path will be used until its energy falls below the energy of the backup path at which the backup path is used. Using this approach, the nodes in the primary path will not deplete their energy resources through continual use of the same route, hence achieving longer life. However, the path switching cost was not quantified in the paper.

The authors of [29] proposed the use of a set of sub-optimal paths occasionally to increase the lifetime of the network. These paths are chosen by means of a probability which depends on how low the energy consumption of each path is.

The path with the largest residual energy when used to route data in a network, may be very energyexpensive too. So, there is a tradeoff between minimizing the total power consumed and the residual energy of the network. The authors in [26] proposed an algorithm in which the residual energy of the route is relaxed a bit in order to select a more energy efficient path. 
In [34], multipath routing was used to enhance the reliability of WSNs. The proposed scheme is useful for delivering data in unreliable environments. It is known that network reliability can be increased by providing several paths from source to destination and by sending the same packet on each path. However, using this technique, traffic will increase significantly. Hence, there is a tradeoff between the amount of traffic and the reliability of the network. This tradeoff is studied in [34] using a redundancy function that is dependent on the multipath degree and on failing probabilities of the available paths. The idea is to split the original data packet into subpackets and then send each subpacket through one of the available multipaths. It has been found that even if some of these subpackets were lost, the original message can still be reconstructed. According to their algorithm, it has also been found that for a given maximum node failure probability, using higher multipath degree than a certain optimal value will increase the total probability of failure.

Directed diffusion [2] is a good candidate for robust multipath routing and delivery. Based on the directed diffusion paradigm, a multipath routing scheme that finds several partially disjoint paths is studied in [10] (alternate routes are not node disjoint, i.e., routes are partially overlapped). It has been found that the use of multipath routing provides viable alternative for energy efficient recovery from failures in WSN. The motivation of using these braided paths is to keep the cost of maintaining the multipaths low. The costs of alternate paths are comparable to the primary path because they tend to be much closer to the primary path.

\subsubsection{Query based routing}

In this kind of routing, the destination nodes propagate a query for data (sensing task) from a node through the network and a node having this data sends the data which matches the query back to the node, which initiates the query. Usually these queries are described in natural language, or in high-level query languages. For example, client $\mathrm{C} 1$ may submit a query to node $\mathrm{N} 1$ and ask: Are there moving vehicles in battle space region 1?. All the nodes have tables consisting of the sensing tasks queries that they receive and send data which matches these tasks when they receive it. Directed diffusion [2] described in Section 3.1.1 is an example of this type of routing. In directed diffusion, the BS node sends out interest messages to sensors. As the interest is propagated throughout the sensor network, the gradients from the source back to the BS are set up. When the source has data for the interest, the source sends the data along the interests gradient path. To lower energy consumption, data aggregation (e.g., duplicate suppression) is performed enroute.

The rumor routing protocol [27] uses a set of long-lived agents to create paths that are directed towards the events they encounter. Whenever an agent crosses path with a path leading to an event that it has not encountered yet, it creates a path state that leads to the event. When the agents come across shorter paths or more efficient paths, they optimize the paths in routing tables accordingly. Each node maintains a list of its neighbors and an events table that is updated whenever new events are encountered. Each node can also generate an agent in a probabilistic fashion. Each agent contains an events table that is synchronized with every node that it visits. The agent has a lifetime of a certain number of hops after which it dies. A node will not generate a query unless it learns a route to the required event. If there is no route available, the node transmit a query in a random direction. Then, node waits to know if the query reached the destination for a certain amount of time, after which the node floods the network if no response is heared from the destination. 


\subsubsection{Negotiation based routing protocols}

These protocols use high level data descriptors in order to eliminate redundant data transmissions through negotiation. Communication decisions are also taken based on the resources that are available to them. The SPIN family protocols [3] discussed earlier and the protocols in [7] are examples of negotiation based routing protocols. The motivation is that the use of flooding to disseminate data will produce implosion and overlap between the sent data, hence nodes will receive duplicate copies of the same data. This operation consumes more energy and more processing by sending the same data by different sensors. The SPIN protocols are designed to disseminate the data of one sensor to all other sensors assuming these sensors are potential base-stations. Hence, the main idea of negotiation based routing in WSNs is to suppress duplicate information and prevent redundant data from being sent to the next sensor or the base-station by conducting a series of negotiation messages before the real data transmission begins.

\subsubsection{QoS-based routing}

In QoS-based routing protocols, the network has to balance between energy consumption and data quality. In particular, the network has to satisfy certain QoS metrics, e.g., delay, energy, bandwidth, etc. when delivering data to the BS.

Sequential Assignment Routing (SAR) proposed in [11] is one of the first routing protocols for WSNs that introduces the notion of QoS in the routing decisions. Routing decision in SAR is dependent on three factors: energy resources, QoS on each path, and the priority level of each packet. To avoid single route failure, a multi-path approach is used and localized path restoration schemes are used. To create multiple paths from a source node, a tree rooted at the source node to the destination nodes (i.e., the set of base-stations (BSs)) is built. The paths of the tree are built while avoiding nodes with low energy or QoS guarantees. At the end of this process, each sensor node will be part of multi-path tree. As such, SAR is table-driven multi-path protocol that aims to achieve energy efficiency and fault tolerance. In essence, SAR calculates a weighted QoS metric as the product of the additive QoS metric and a weight coefficient associated with the priority level of the packet. The objective of SAR algorithm is to minimize the average weighted QoS metric throughout the lifetime of the network. If topology changes due to node failures, a path re-computation is needed. As a preventive measure, a periodic re-computation of paths is triggered by the base-station to account for any changes in the topology. A handshake procedure based on a local path restoration scheme between neighboring nodes is used to recover from a failure. Failure recovery is done by enforcing routing table consistency between upstream and downstream nodes on each path. Simulation results showed that SAR offers less power consumption than the minimum-energy metric algorithm, which focuses only the energy consumption of each packet without considering its priority. SAR maintains multiple paths from nodes to BS. Although, this ensures fault-tolerance and easy recovery, the protocol suffers from the overhead of maintaining the tables and states at each sensor node especially when the number of nodes is huge.

Another QoS routing protocol for WSNs that provides soft real-time end-to-end guarantees was introduced in [44]. The protocol requires each node to maintain information about its neighbors and uses geographic forwarding to find the paths. In addition, SPEED strive to ensure a certain speed for each packet in the network so that each application can estimate the end-to-end delay for the packets by dividing the distance to the BS by the speed of the packet before making the admission decision. Moreover, 
SPEED can provide congestion avoidance when the network is congested. The routing module in SPEED is called Stateless Geographic Non-Deterministic forwarding (SNFG) and works with four other modules at the network layer. Delay estimation at each node is basically made by calculating the elapsed time when an ACK is received from a neighbor as a response to a transmitted data packet. By looking at the delay values, SNGF selects the node, which meets the speed requirement. If it fails, the relay ratio of the node is checked, which is calculated by looking at the miss ratios of the neighbors of a node (the nodes which could not provide the desired speed) and is fed to the SNGF module. When compared to Dynamic Source Routing (DSR) and Ad-hoc on-demand vector routing, SPEED performs better in terms of end-to-end delay and miss ratio. Moreover, the total transmission energy is less due to the simplicity of the routing algorithm, i.e., control packet overhead is less. However, SPEED does not consider any further energy metric in its routing protocol. Therefore, for more realistic understanding of SPEED's energy consumption, there is a need to compare it to a routing protocol that is energy-aware.

\subsubsection{Coherent and non-coherent processing}

Data processing is a major component in the operation of wireless sensor networks. Hence, routing techniques employ different data processing techniques. In general, sensor nodes will cooperate with each other in processing different data flooded in the network area. Two examples of data processing techniques proposed in WSNs are coherent and non-coherent data processing-based routing [11]. In non-coherent data processing routing, nodes will locally process the raw data before being sent to other nodes for further processing. The nodes that perform further processing are called the aggregators. In coherent routing, the data is forwarded to aggregators after minimum processing. The minimum processing typically includes tasks like time stamping, duplicate suppression, etc. To perform energy-efficient routing, coherent processing is normally selected.

Non-coherent functions have fairly low data traffic loading. On the other hand, since coherent processing generates long data streams, energy efficiency must be achieved by path optimality. In non-coherent processing, data processing incurs three phases: (1) Target detection, data collection, and preprocessing (2) Membership declaration, and (3) Central node election. During phase 1, a target is detected, its data collected and preprocessed. When a node decides to participate in a cooperative function, it will enter phase 2 and declare this intention to all neighbors. This should be done as soon as possible so that each sensor has a local understanding of the network topology. Phase 3 is the election of the central node. Since the central node is selected to perform more sophisticated information processing, it must have sufficient energy reserves and computational capability.

In [11], a single and multiple winner algorithms were proposed for non-coherent and coherent processing, respectively. In the single winner algorithm (SWE), a single aggregator node is elected for complex processing. The election of a node is based on the energy reserves and computational capability of that node. By the end of the SWE process, a minimum-hop spanning tree will completely cover the network. In the multiple winner algorithm (MWE), a simple extension to the single winner algorithm (SWE) is proposed. When all nodes are sources and send their data to the central aggregator node, a large amount of energy will be consumed and hence this process has a high cost. One way to lower the energy cost is to limit the number of sources that can send data to the central aggregator node. Instead of keeping record of only the best candidate node (master aggregator node), each node will keep a record of up to $n$ nodes of those candidates. At the end of the MWE process, each sensor in the network has a set of 
minimum-energy paths to each source node (SN). After that, the single winner algorithm is used to find the node that yields the minimum energy consumption. This node can then serves as the central node for the coherent processing. In general, the MWE process has longer delay, higher overhead, and lower scalability than that for non-coherent processing networks.

We observed that there are some hybrid protocols that fit under more than one category. We summarize recent research results on data routing in WSNs in the Table shown in Figure 8. The Table shows how different routing protocols fit under different category and also compare different routing techniques according to many metrics.

\begin{tabular}{|c|c|c|c|c|c|c|c|c|c|c|c|c|}
\hline & \begin{tabular}{|l|} 
Classification \\
\end{tabular} & \begin{tabular}{|l|} 
Mobility \\
\end{tabular} & \begin{tabular}{|l|} 
Position \\
Awareness
\end{tabular} & $\begin{array}{l}\text { Power } \\
\text { Usage }\end{array}$ & $\begin{array}{l}\text { Negotiation } \\
\text { based }\end{array}$ & \begin{tabular}{|l|} 
Data \\
Aggregation
\end{tabular} & Localization & QoS & \begin{tabular}{|l|} 
State \\
Complexity
\end{tabular} & Scalability & Multipath & Query based \\
\hline SPIN & Flat & \begin{tabular}{|l|} 
Possible \\
\end{tabular} & \begin{tabular}{|l} 
No \\
\end{tabular} & Limited & Yes & Yes & No & No & Low & Limited & Yes & Yes \\
\hline $\begin{array}{l}\text { Directed } \\
\text { Diffusion }\end{array}$ & Flat & Limited & No & Limited & Yes & Yes & Yes & No & Low & Limited & Yes & Yes \\
\hline $\begin{array}{l}\text { Rumor } \\
\text { Routing }\end{array}$ & Flat & \begin{tabular}{|l|} 
Very \\
Limited
\end{tabular} & No & N/A & No & Yes & No & No & Low & Good & No & Yes \\
\hline GBR & Flat & Limited & No & N/A & No & Yes & No & No & Low & Limited & No & Yes \\
\hline MCFA & Flat & No & No & N/A & No & No & No & No & Low & Good & No & No \\
\hline CADR & Flat & No & No & Limited & No & Yes & No & No & Low & Limited & No & No \\
\hline COUGAR & Flat & No & No & Limited & No & Yes & No & No & Low & Limited & No & Yes \\
\hline ACQUIRE & Flat & Limited & No & N/A & No & Yes & No & No & Low & Limited & No & Yes \\
\hline EAR & Flat & Limited & No & N/A & No & No & & No & Low & Limited & No & Yes \\
\hline LEACH & \begin{tabular}{|l} 
Hierarchical \\
\end{tabular} & \begin{tabular}{|l|} 
Fixed BS \\
\end{tabular} & No & Maximum & No & Yes & Yes & No & CHs & Good & No & No \\
\hline $\begin{array}{l}\text { TEEN \& } \\
\text { APTEEN }\end{array}$ & Hierarchical & Fixed BS & No & Maximum & No & Yes & Yes & No & CHs & Good & No & No \\
\hline PEGASIS & Hierarchical & \begin{tabular}{|l|} 
Fixed BS \\
\end{tabular} & No & Maximum & No & No & Yes & \begin{tabular}{|l|} 
No \\
\end{tabular} & Low & Good & No & No \\
\hline $\begin{array}{l}\text { MECN \& } \\
\text { SMECN }\end{array}$ & Hierarchical & No & No & Maximum & No & No & No & No & Low & Low & No & No \\
\hline SOP & \begin{tabular}{|l} 
Hierarchical \\
\end{tabular} & No & No & N/A & No & \begin{tabular}{|l|} 
No \\
\end{tabular} & No & No & Low & Low & No & No \\
\hline HPAR & \begin{tabular}{|l} 
Hierarchical \\
\end{tabular} & No & No & N/A & No & No & No & No & Low & Good & No & No \\
\hline VGA & Hierarchical & No & No & N/A & Yes & Yes & Yes & No & CHs & Good & Yes & No \\
\hline $\begin{array}{l}\text { Sensor } \\
\text { aggregate }\end{array}$ & Hierarchical & Limited & No & N/A & No & Yes & No & No & Low & Good & No & Possible \\
\hline TTDD & Hierarchical & Yes & Yes & Limited & No & No & No & No & Moderate & Low & \begin{tabular}{|l|} 
Possible \\
\end{tabular} & \begin{tabular}{|l|} 
Possible \\
\end{tabular} \\
\hline GAF & \begin{tabular}{|l|} 
Location \\
\end{tabular} & Limited & No & Limited & No & No & No & No & Low & Good & No & No \\
\hline GEAR & \begin{tabular}{|l|} 
Location \\
\end{tabular} & Limited & No & Limited & No & No & No & No & Low & Limited & No & No \\
\hline SPAN & \begin{tabular}{|l|} 
Location \\
\end{tabular} & Limited & No & N/A & Yes & No & No & No & Low & Limited & No & No \\
\hline $\begin{array}{l}\text { MFR, } \\
\text { GEDIR }\end{array}$ & Location & No & No & N/A & No & No & No & No & Low & Limited & No & No \\
\hline GOAFR & Location & No & No & N/A & No & No & No & & Low & Good & No & No \\
\hline SAR & QoS & No & No & N/A & Yes & Yes & No & Yes & \begin{tabular}{|l|} 
Moderate \\
\end{tabular} & Limited & No & Yes \\
\hline SPEED & QoS & No & No & N/A & No & \begin{tabular}{|l|} 
No \\
\end{tabular} & No & Yes & moderate & Limited & No & Yes \\
\hline
\end{tabular}

Figure 8: Classification and comparison of routing protocols in wireless sensor networks

\section{Routing in WSNs: Future Directions}

The future vision of WSNs is to embed numerous distributed devices to monitor and interact with physical world phenomena, and to exploit spatially and temporally dense sensing and actuation capabilities of those sensing devices. These nodes coordinate among themselves to create a network that performs higher-level tasks.

Although extensive efforts have been exerted so far on the routing problem in WSNs, there are still some challenges that confront effective solutions of the routing problem. First, there is a tight coupling between sensor nodes and the physical world. Sensors are embedded in unattended places or systems. This is different from traditional Internet, PDA, and mobility applications that interface primarily and directly with human users. Second, sensors are characterized by a small foot print, and as such nodes present stringent energy constraints since they are equipped with small, finite, energy source. This is also different from traditional fixed but reusable resources. Third, communications is primary consumer of energy in this environment where sending a bit over 10 or 100 meters consumes as much energy as thousands-to-millions of operations (known as $R^{4}$ signal energy drop-off) [36]. 
Although the performance of these protocols is promising in terms of energy efficiency, further research would be needed to address issues such as Quality of Service (QoS) posed by video and imaging sensors and real-time applications. Energy-aware QoS routing in sensor networks will ensure guaranteed bandwidth (or delay) through the duration of connection as well as providing the use of most energy efficient path. Another interesting issue for routing protocols is the consideration of node mobility. Most of the current protocols assume that the sensor nodes and the BS are stationary. However, there might be situations such as battle environments where the BS and possibly the sensors need to be mobile. In such cases, the frequent update of the position of the command node and the sensor nodes and the propagation of that information through the network may excessively drain the energy of nodes. New routing algorithms are needed in order to handle the overhead of mobility and topology changes in such energy constrained environment. Future trends in routing techniques in WSNs focus on different directions, all share the common objective of prolonging the network lifetime. We summarize some of these directions and give some pertinent references as follows:

- Exploit redundancy: typically a large number of sensor nodes are implanted inside or beside the phenomenon. Since sensor nodes are prone to failure, fault tolerance techniques come in picture to keep the network operating and performing its tasks. Routing techniques that explicitly employ fault tolerance techniques in an efficient manner are still under investigation (e.g., [34]).

- Tiered architectures (mix of form/energy factors): Hierarchical routing is an old technique to enhance scalability and efficiency of the routing protocol. However, novel techniques to network clustering which maximize the network lifetime are also a hot area of research in WSNs (e.g., [45]).

- Exploit spatial diversity and density of sensor/actuator nodes: Nodes will span a network area that might be large enough to provide spatial communication between sensor nodes. Achieving energy efficient communication in this densely populated environment deserves further investigation. The dense deployment of sensor nodes should allow the network to adapt to unpredictable environment.

- Achieve desired global behavior with adaptive localized algorithms (i.e., do not rely on global interaction or information). However, in a dynamic environment, this is hard to model (e.g., [2]).

- Leverage data processing inside the network and exploit computation near data sources to reduce communication, i.e., perform in-network distributed processing. WSNs are organized around naming data, not nodes identities. Since we have a large collections of distributed elements, localized algorithms that achieve system-wide properties in terms of local processing of data before being sent to the destination are still needed. Nodes in the network will store named data and make it available for processing. There is a high need to create efficient processing points in the network, e.g., duplicate suppression, aggregation, correlation of data. How to efficiently and optimally find those points is still an open research issue (e.g., [31]).

- Time and location synchronization: energy-efficient techniques for associating time and spatial coordinates with data to support collaborative processing are also required [20].

- Localization: sensor nodes are randomly deployed into an unplanned infrastructure. The problem of estimating spatial-coordinates of the node is referred to as localization. Global Positioning System (GPS) cannot be used in WSNs as GPS can work only outdoors and cannot work in the presence of 
any obstruction. Moreover, GPS receivers are expensive and not suitable in the construction of small cheap sensor nodes. Hence, there is a need to develop other means of establishing a coordinate system without relying on an existing infrastructure. Most of the proposed localization techniques today, depend on recursive trilateration/multilateration techniques (e.g., [38]) which would not provide enough accuracy in WSNs.

- Self-configuration and reconfiguration is essential to lifetime of unattended systems in dynamic, and constrained energy environment. This is important for keeping the network up and running. As nodes die and leave the network, update and reconfiguration mechanisms should take place. A feature that is important in every routing protocol is to adapt to topology changes very quickly and to maintain the network functions (e.g., [3]).

- Secure Routing: Current routing protocols optimize for the limited capabilities of the nodes and the application specific nature of the networks, but do not consider security. Although these protocols have not been designed with security as a goal, it is important to analyze their security properties. One aspect of sensor networks that complicates the design of a secure routing protocol is in-network aggregation. In WSNs, in-network processing makes end-to-end security mechanisms harder to deploy because intermediate nodes need direct access to the contents of the messages (e.g., [5], [40]).

- Other possible future research for routing protocols includes the integration of sensor networks with wired networks (i.e. Internet). Most of the applications in security and environmental monitoring require the data collected from the sensor nodes to be transmitted to a server so that further analysis can be done. On the other hand, the requests from the user should be made to the BS through Internet. Since the routing requirements of each environment are different, further research is necessary for handling these kinds of situations.

\section{Conclusions}

Routing in sensor networks is a new area of research, with a limited, but rapidly growing set of research results. In this paper, we presented a comprehensive survey of routing techniques in wireless sensor networks which have been presented in the literature. They have the common objective of trying to extend the lifetime of the sensor network, while not compromising data delivery.

Overall, the routing techniques are classified based on the network structure into three categories: flat, hierarchical, and location based routing protocols. Furthermore, these protocols are classified into multipath-based, query-based, negotiation-based, or QoS-based routing techniques depending on the protocol operation. We also highlight the design tradeoffs between energy and communication overhead savings in some of the routing paradigm, as well as the advantages and disadvantages of each routing technique. Although many of these routing techniques look promising, there are still many challenges that need to be solved in the sensor networks. We highlighted those challenges and pinpointed future research directions in this regard. 


\section{References}

[1] W. Heinzelman, A. Chandrakasan and H. Balakrishnan, "Energy-Efficient Communication Protocol for Wireless Microsensor Networks," Proceedings of the 33rd Hawaii International Conference on System Sciences (HICSS '00), January 2000.

[2] C. Intanagonwiwat, R. Govindan, and D. Estrin, "Directed diffusion: a scalable and robust communication paradigm for sensor networks," Proceedings of ACM MobiCom '00, Boston, MA, 2000, pp. 56-67.

[3] W. Heinzelman, J. Kulik, and H. Balakrishnan, "Adaptive Protocols for Information Dissemination in Wireless Sensor Networks," Proc. 5th ACM/IEEE Mobicom Conference (MobiCom '99), Seattle, WA, August, 1999. pp. 174-85.

[4] I. Akyildiz, W. Su, Y. Sankarasubramaniam, and E. Cayirci, "A survey on sensor networks," IEEE Communications Magazine, Volume: 40 Issue: 8, pp.102-114, August 2002.

[5] A. Perrig, R. Szewzyk, J.D. Tygar, V. Wen, and D. E. Culler, "SPINS: security protocols for sensor networks". Wireless Networks Volume: 8, pp. 521-534, 2000.

[6] S. Hedetniemi and A. Liestman, "A survey of gossiping and broadcasting in communication networks", IEEE Networks, Vol. 18, No. 4, pp. 319-349, 1988.

[7] J. Kulik, W. R. Heinzelman, and H. Balakrishnan, "Negotiation-based protocols for disseminating information in wireless sensor networks," Wireless Networks, Volume: 8, pp. 169-185, 2002.

[8] A. Manjeshwar and D. P. Agarwal, "TEEN: a routing protocol for enhanced efficiency in wireless sensor networks," In 1st International Workshop on Parallel and Distributed Computing Issues in Wireless Networks and Mobile Computing, April 2001.

[9] A. Manjeshwar and D. P. Agarwal, "APTEEN: A hybrid protocol for efficient routing and comprehensive information retrieval in wireless sensor networks," Parallel and Distributed Processing Symposium., Proceedings International, IPDPS 2002, pp. 195-202.

[10] D. Ganesan, R. Govindan, S. Shenker, and D. Estrin, "Highly-resilient, energy-efficient multipath routing in wireless sensor networks", ACM SIGMOBILE Mobile Computing and Communications Review, vol. 5, no. 4, pp. $1125,2001$.

[11] K. Sohrabi, J. Pottie, "Protocols for self-organization of a wireless sensor network", IEEE Personal Communications, Volume 7, Issue 5, pp 16-27, 2000.

[12] L. Subramanian and R. H. Katz, "An Architecture for Building Self Configurable Systems", in the Proceedings of IEEE/ACM Workshop on Mobile Ad Hoc Networking and Computing, Boston, MA, August 2000.

[13] Y. Yao and J. Gehrke, "The cougar approach to in-network query processing in sensor networks", in SIGMOD Record, September 2002.

[14] D. Braginsky and D. Estrin, "Rumor Routing Algorithm for Sensor Networks," in the Proceedings of the First Workshop on Sensor Networks and Applications (WSNA), Atlanta, GA, October 2002.

[15] ] C. Schurgers and M.B. Srivastava, "Energy efficient routing in wireless sensor networks", in the MILCOM Proceedings on Communications for Network-Centric Operations: Creating the Information Force, McLean, VA, 2001.

[16] M. Chu, H. Haussecker, and F. Zhao, "Scalable Information-Driven Sensor Querying and Routing for ad hoc Heterogeneous Sensor Networks," The International Journal of High Performance Computing Applications, Vol. 16, No. 3, August 2002 .

[17] S. Lindsey, C. Raghavendra, "PEGASIS: Power-Efficient Gathering in Sensor Information Systems", IEEE Aerospace Conference Proceedings, 2002, Vol. 3, 9-16 pp. 1125-1130.

[18] F. Ye, A. Chen, S. Liu, L. Zhang, "A scalable solution to minimum cost forwarding in large sensor networks", Proceedings of the tenth International Conference on Computer Communications and Networks (ICCCN), pp. 304-309, 2001.

[19] F. Ye, H. Luo, J. Cheng, S. Lu, L. Zhang, "A Two-tier data dissemination model for large-scale wireless sensor networks", proceedings of ACM/IEEE MOBICOM, 2002.

[20] N. Bulusu, J. Heidemann, D. Estrin, "GPS-less low cost outdoor localization for very small devices", Technical report 00-729, Computer science department, University of Southern California, Apr. 2000.

[21] A. Savvides, C-C Han, aind M. Srivastava, "Dynamic fine-grained localization in Ad-Hoc networks of sensors," Proceedings of the Seventh ACM Annual International Conference on Mobile Computing and Networking (MobiCom), July 2001. pp. $166-179$.

[22] V. Rodoplu and T. H. Meng, "Minimum Energy Mobile Wireless Networks", IEEE Journal Selected Areas in Communications, vol. 17, no. 8, Aug. 1999, pp. 133344.

[23] L. Li, and J. Y. Halpern, "Minimum-Energy Mobile Wireless Networks Revisited," IEEE International Conference on Communications (ICC) 2001. Vol. 1, pp. 278-283.

[24] S. Hedetniemi, S. Hedetniemi, and A. Liestman, "A Survey of Gossiping and Broadcasting in Communication Networks," Networks, vol. 18, 1988. 
[25] Y. Xu, J. Heidemann, D. Estrin, "Geography-informed Energy Conservation for Ad-hoc Routing," In Proceedings of the Seventh Annual ACM/IEEE International Conference on Mobile Computing and Networking 2001, pp. 70-84.

[26] Q. Li and J. Aslam and D. Rus, "Hierarchical Power-aware Routing in Sensor Networks", In Proceedings of the DIMACS Workshop on Pervasive Networking, May, 2001.

[27] D. Braginsky and D. Estrin, "Rumor Routing Algorithm For Sensor Networks", International Conference on Distributed Computing Systems (ICDCS'01), November 2001.

[28] J.-H. Chang and L. Tassiulas, "Maximum Lifetime Routing in Wireless Sensor Networks", Proc. Advanced Telecommunications and Information Distribution Research Program (ATIRP2000), College Park, MD, Mar. 2000.

[29] C. Rahul, J. Rabaey, "Energy Aware Routing for Low Energy Ad Hoc Sensor Networks", IEEE Wireless Communications and Networking Conference (WCNC), vol.1, March 17-21, 2002, Orlando, FL, pp. 350-355.

[30] S. Capkun, M. Hamdi, J. Hubaux,"GPS-free positioning in mobile ad-hoc networks", Proceedings of the 34th Annual Hawaii International Conference on System Sciences, 2001 pp. 3481-3490.

[31] Jamal N. Al-Karaki, Raza Ul-Mustafa, Ahmed E. Kamal, "Data Aggregation in Wireless Sensor Networks - Exact and Approximate Algorithms"', Proceedings of IEEE Workshop on High Performance Switching and Routing (HPSR) 2004, April 18-21, 2004, Phoenix, Arizona, USA.

[32] http://www.ieee802.org/15/

[33] B. Chen, K. Jamieson, H. Balakrishnan, R. Morris, "SPAN: an energy-efficient coordination algorithm for topology maintenance in ad hoc wireless networks", Wireless Networks, Vol. 8, No. 5, Page(s): 481-494, September 2002.

[34] S. Dulman, T. Nieberg, J. Wu, P. Havinga, "Trade-Off between Traffic Overhead and Reliability in Multipath Routing for Wireless Sensor Networks", WCNC Workshop, New Orleans, Louisiana, USA, March 2003.

[35] Q. Fang, F. Zhao, and L. Guibas, "Lightweight Sensing and Communication Protocols for Target Enumeration and Aggregation", Proceedings of the 4th ACM international symposium on Mobile ad hoc networking and computing (MOBIHOC), 2003, pp. 165-176.

[36] D. Goodman, "Wireless Personal Communications Systems". Reading, MA: Addison-Wesley, 1997.

[37] S. Tilak, N. Abu-Ghazaleh, W. Heinzelman, "A taxonomy of wireless micro-sensor network models", ACM SIGMOBILE Mobile Computing and Communications Review, Volume 6, Issue 2 (April 2002), pp 28-36.

[38] N. Bulusu, D. Estrin, L. Girod and J. Heidemann, "Scalable Coordination for wireless sensor networks: Self-Configuring Localization Systems", In Proceedings of the Sixth International Symposium on Communication Theory and Applications (ISCTA 2001), Ambleside, Lake District,UK, July 2001

[39] R. C. Shah and J. Rabaey, "Energy Aware Routing for Low Energy Ad Hoc Sensor Networks", IEEE Wireless Communications and Networking Conference (WCNC), March 17-21, 2002, Orlando, FL.

[40] C. Karlof, D. Wagner, "Secure routing in wireless sensor networks: attacks and countermeasures", Ad Hoc Networks, Vol. 1 (2003) pp. 293315.

[41] N. Sadagopan et al., The ACQUIRE mechanism for efficient querying in sensor networks, in the Proceedings of the First International Workshop on Sensor Network Protocol and Applications, Anchorage, Alaska, May 2003.

[42] Y. Yu, D. Estrin, and R. Govindan, "Geographical and Energy-Aware Routing: A Recursive Data Dissemination Protocol for Wireless Sensor Networks", UCLA Computer Science Department Technical Report, UCLA-CSD TR-01-0023, May 2001.

[43] B. Karp and H. T. Kung, "GPSR: Greedy perimeter stateless routing for wireless sensor networks", in the Proceedings of the 6th Annual ACM/IEEE International Conference on Mobile Computing and Networking (MobiCom '00), Boston, MA, August 2000.

[44] T. He et al., "SPEED: A stateless protocol for real-time communication in sensor networks", in the Proceedings of International Conference on Distributed Computing Systems, Providence, RI, May 2003.

[45] S. Bandyopadhyay, E. Coyle, "An Energy Efficient Hierarchical Clustering Algorithm for Wireless Sensor Networks", Proceedings of INFOCOM 2003, Vol. 3, pp. 1713-1723.

[46] I. Stojmenovic and X. Lin. "GEDIR: Loop-Free Location Based Routing in Wireless Networks", In International Conference on Parallel and Distributed Computing and Systems, Boston, MA, USA, Nov. 3-6, 1999.

[47] F. Kuhn, R. Wattenhofer, A. Zollinger, "Worst-Case optimal and average-case efficient geometric ad-hoc routing", Proceedings of the 4th ACM International Conference on Mobile Computing and Networking, Pages: 267-278, 2003.

[48] Jamal N. Al-Karaki, A.E. Kamal, "On the Correlated Data Gathering Problem in Wireless Sensor Networks", to appear in the Proceedings of The Ninth IEEE Symposium on Computers and Communications, Alexandria, Egypt, July 2004

[49] S. Servetto and G. Barrenechea, "Constrained Random Walks on Random Graphs: Routing Algorithms for Large Scale Wireless Sensor Networks", proceedings of the 1st ACM International Workshop on Wireless Sensor Networks and Applications, Atlanta, Georgia, USA, 2002. 